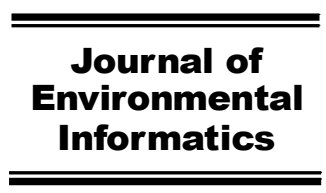

www.iseis.org/jei

\title{
Interval Recourse Linear Programming for Resources and Environmental Systems Management under Uncertainty
}

\author{
G. H. Cheng ${ }^{1}$, G. H. Huang, ${ }^{2,}$, C. Dong ${ }^{1}$, B. W. Baetz ${ }^{3}$, and Y. P. Li ${ }^{4}$ \\ ${ }^{I}$ Center for Energy, Environment and Ecology Research, UR-BNU, Faculty of Engineering and Applied Science, University of Regina, Regina, \\ Saskatchewan S4S OA2, Canada \\ ${ }^{2}$ Center for Energy, Environment and Ecology Research, UR-BNU, Beijing Normal University, Beijing 100875, China \\ ${ }^{3}$ Faculty of Engineering, McMaster University, Ontario L8S 4L8, Canada \\ ${ }^{4}$ State Key Laboratory of Water Environment Simulation, School of Environment, Beijing Normal University, Beijing 100875, China
}

Received 28 June 2014; revised 23 September 2014; accepted 10 December 2014; published online 8 February 2015

\begin{abstract}
Interval recourse linear programming $(I R L P)$ is proposed for mitigating constraint violation problems in resources and environmental systems management $(R E M)$ under interval uncertainties. Based on a review of interval linear programming $(I L P)$ and its significances to $R E M$, two linear programming sub-models are employed to initialize a decision space in $I R L P$. The causes of constraint violation are examined based on identification of a violation criterion. Contraction ratios are defined after revelation of violation ranges of constraints. As a recourse measure to resolve constraint violation problems, another two linear programming sub-models are constructed given a series of contraction ratios. A hypercube decision space where infeasible solutions are excluded is obtained. A postoptimality analysis is conducted to deal with the barriers for applying the IRLP approach to address real-world REM problems under interval uncertainties. A selected REM problem is introduced to demonstrate the procedures and effectiveness of the IRLP approach. Comparisons with existing ILP methods reveal that the IRLP approach is effective at avoiding constraint violation, reproducing the largest decision space which does not include infeasible solutions, and enhancing the reliability of decision support for REM.
\end{abstract}

Keywords: resources and environmental management; interval linear programming; constraint violation

\section{Introduction}

It is common that properties of elements or influencing factors of resources and environmental management (REM) systems are of interval uncertainties. Effective reflection of this system complexity in the decision support process is desired for assuring reliability of suggested management schemes and avoiding penalties to socio-economic and eco-environmental profits. One of representative approaches for achieving it is interval linear programming $(I L P)$. In the past decades, a number of $I L P$ models which were mixtures of linear programming models and interval-set coefficients were developed (e.g., Soyster, 1973; Moore, 1979; Steuer, 1981; Huang et al., 1992; Sengupta and Pal, 2000; Sengupta et al., 2001; Hladik, 2012). Among them, the first ILP model that could reflect multiplicities of decision alternatives under interval uncertainties was developed by Huang et al. (1992). Due to higher robustness than others in terms of approaching realities, it has been applied to many realworld REM problems such as waste management (Chang et al., 1997; Pires et al., 2011; Wu et al, 2015), water resources alloca-

* Corresponding author. Tel.: +1 306 5854095; fax: +1 3063373205 .

Email address: huang@iseis.org (G. H. Huang).

ISSN: $1726-2135$ print/1684-8799 online

(C) 2017 ISEIS All rights reserved. doi:10.3808/jei.201500312 tion (Maqsood et al., 2005; Li et al., 2008; Lv et al., 2010; Nikoo et al., 2012), energy systems management (Lin et al., 2005; Cai et al., 2009; Dong et al., 2012; Li et al., 2014), and water quality management (Zeng et al., 2000; Guo et al., 2003; Jin et al., 2004; Qin et al., 2009; Nikoo et al., 2012; Hu et al., 2013; Liu et al., 2015). However, the effectiveness of the ILP method (Huang et al., 1992) is dependent with solution methods.

Previously, many methods (e.g., Charnes et al., 1977; Ishibuchi and Tanaka, 1990; Huang et al., 1992; Tong, 1994; Inuiguchi and Sakawa, 1995, 1997; Chanas and Kuchta, 1996; Sengupta and Pal, 2000; Chinneck and Ramadan, 2000; Ben-Tal and Nemirovski, 2002; Inuiguchi et al., 2003; Bertsimas and Sim, 2004; Ben-Tal et al., 2009; Bertsimas and Brown, 2011; Gabrel et al., 2014) were developed for solving the ILP model (Huang et al., 1992). For instance, an ILP model could be equivalent to a robust linear programming $(R L P)$ problem; a robust solution of which performances remained relatively unchanged when exposed to uncertain conditions (Beyer and Sendhoff, 2007) could be obtained from a robust counterpart of the $R L P$ model. Tong (1994), Levin (1994) and Chinneck and Ramadan (2000) proposed methods that could obtain the most optimistic decision scheme from a best-case sub-model and the most conservative one from a worst-case sub-model. These methods were challenged by lack of abundant alternatives for decision making under interval uncertainties. To mitigate this challenge, Huang et al. (1992) proposed a two-step approach (TSA) in which a continuous decision space could be provided for decision makers based on two correlated linear programming sub-mo- 
dels (denoted as $I L P 1$ and ILP2).

Zhou et al. (2008) pointed out that constraints might be violated by solutions in the decision space for TSA. Accordingly, a modified interval linear programming (MILP) method, a three-step $(T h S)$ method and a robust two-step (RTS) method were contributed by Zhou et al. (2008), Huang and Cao (2011) and Fan and Huang (2012), respectively, to deal with such a difficulty and eliminate unexpected infeasibilities. In MILP, an additional constraint was coupled with constraints of sub-model ILP2 for avoiding violation of constraints. In the $T h S$ method, another sub-model was constructed to constrict the initial decision space obtained from sub-models $I L P 1$ and $I L P 2$. Parameters constricting ratios of decision variables were introduced to reflect constricting ranges of initialized interval-set solutions. They were optimized through the third sub-model subject to avoidance of constraint violation. In the RTS method, submodel ILP2 that focused on conservative schemes was solved prior to sub-model ILP1. A group of additional constraints was incorporated into the original sub-model ILPI for avoiding occurrence of constraint violation.

However, the constraint-violation problem of the TSA approach is not effectively resolved yet. Methods of MILP, ThS and RTS cannot reproduce the largest decision space which does not include infeasible solutions. Part of decision alternatives are neglected due to ineffectiveness of these methods. As one of representative strengthens of the TSA approach, a hypercube decision space that covers most alternative schemes for ILP problems under interval uncertainties can be identified. Failures to remain such strengthen as well as others may reduce reliability of optimization efforts, neglect desired schemes for decision makers of diverse preferences, and sacrifice potential optimality of real-world REM systems.

Therefore, an interval recourse linear programming (IRLP) approach will be proposed in this study for mitigating constraint violation and enhancing reliability of decision support for $R E M$ under interval uncertainties. In Section 2, a review of ILP models, the TSA approach and their significances to REM problems will be conducted. As the first stage of $I R L P$, two linear programming sub-models ILPI and ILP2 in TSA will be employed to initialize a decision space. Causes of constraint violation will be examined based on identification of a violation criterion in Section 3. Contraction ratios will be defined after revelation of violation ranges of constraints. As a recourse measure to constraint violation problems, two linear programming submodels (ILP3 and ILP4) will be constructed in Section 4 given a series of contraction ratios. A hypercube decision space where infeasible solutions are excluded through sub-models ILP3 and ILP4 will be obtained for decision makers. Post-optimality analysis in Section 5 will help enhance adaptability of the IRLP approach to interval linear REM problems of various complexities. In Section 6, an REM problem that can be characterized as an ILP model will be introduced to demonstrate procedures and effectiveness of the proposed IRLP approach. Strengthens of the IRLP approach for REM problems will be revealed from comparisons with existing ILP methods in Section 7. Its shortcomings and potential solution strategies will also be examined.

\section{Review of Interval Linear Programming}

\subsection{Characterization of REM systems}

Properties of elements or influencing factors in real-world $R E M$ systems may be of uncertainties due to ineffectiveness of estimation techniques, inaccuracy of data monitoring, vagueness of subjective evaluation, unpredictability of system noises, or other potential causes. One of possible cases is that the only reliable estimation of these properties is a series of fluctuation ranges of which the distributional information is unknown. For example, rates of nitrogen use in Canada ranged from 25 to 225 $\mathrm{kg} / \mathrm{ha}$ in 2000 (http://www.fao.org), and the growth of energy demand in Canada will range from 0.3 to $1.4 \%$ per year as estimated by the National Energy Board (http://www.neb-one.gc.c a/index-eng.html).

These properties of interval uncertainties can be expressed as interval sets (Huang et al., 1992) that are defined as closed and bounded sets of real numbers. One representative characteristic is that the distributional information is unknown for any real number in interval sets. Reflection of these properties in other forms, e.g., real numbers, random variables, fuzzy sets or their combinations, would decrease robustness of the constructed programming model. Simplification of interval sets into constants may lead to loss of valuable information (i.e., boundaries of properties). Other uncertainty analysis methods such as stochastic analysis and fuzzy set theory can barely reflect these properties. Artificially assumed information that does not exist would be adulterated into the optimization process if interval uncertainties are interpreted as random variables with probability density functions, fuzzy sets with membership functions, or more complex forms such as random fuzzy sets or fuzzy random variables.

Coupling interval sets with linear programming models leads to a range of interval linear programming (ILP) models (Steuer, 1981; Huang et al., 1992; Sengupta and Pal, 2000; Sengupta et al., 2001; Hladık, 2012). Coefficients in both the objective function and constraints of ILP models can be interval sets. It is of low reliability that a deterministic solution is provided for decision making under interval uncertainties. The solution can hardly reflect the trade-off between system optimality and constraint-violation risks. A solution which is a set of interval sets is desired for ILP problems. Accordingly, a generalized ILP model is an $L P$ model where both coefficients and decision variables are interval sets. The first generalized ILP model is proposed by Huang et al. (1992) based on interval analysis. According to (Huang et al., 1992), an ILP model can be formulated as:

$\operatorname{Max} F^{ \pm}=\boldsymbol{C}^{ \pm} \boldsymbol{X}^{ \pm}$

subject to

$\boldsymbol{A}^{ \pm} \boldsymbol{X}^{ \pm} \leq \boldsymbol{b}^{ \pm}$

$\boldsymbol{X}^{ \pm} \geq \mathbf{0}$

where $\boldsymbol{X}^{ \pm}=\left\{x_{j}^{ \pm}\right\}^{n \times 1}, \boldsymbol{C}^{ \pm}=\left\{c_{j}^{ \pm}\right\}^{1 \times n}, \boldsymbol{A}^{ \pm}=\left\{a_{i j}^{ \pm}\right\}^{m \times n}, \boldsymbol{b}^{ \pm}=\left\{b_{i}^{ \pm}\right\}^{m \times 1}$; 
positive integers $n$ and $m$ are numbers of decision variables $\left\{x_{j}^{ \pm}\right\}$and constraints (1-2), respectively; for any $i \in\{1,2, \ldots$, $m\}$ and $j \in\{1,2, \ldots, n\}$, coefficients $c_{j}^{ \pm}, a_{i j}{ }^{ \pm}$and $b_{i}{ }^{ \pm}$are interval sets of which values range from a real-valued lower bound to a real-valued upper bound without distribution information $(\mathrm{Hu}-$ ang et al., 1992); the lower and upper bounds of interval sets are denoted as symbols ${ }^{-}$and $^{+}$, respectively; decision variables $\left\{x_{j}^{ \pm} \mid j=1,2, \ldots, n\right\}$ as well as the objective function value $\left(F^{ \pm}\right)$ are also interval sets due to interval uncertainties of coefficients; $\mathbf{0}=\{0\}^{n \times 1}$; and the programming objective is to maximize the value range of objective function $F^{ \pm}$.

\subsection{Principle of $I L P$ Solution Algorithm}

Suppose $s^{ \pm}=\left[s^{-}, s^{+}\right]$be any interval set. Any real value belonging to $s^{ \pm}$, e.g., $s \in\left[s^{-}, s^{+}\right]$, is defined as a whitened value of $s^{ \pm}$(Huang et al., 1992). Any interval-set coefficient (e.g., $c_{j}^{ \pm}, a_{i j}{ }^{ \pm}$ or $b_{i}^{ \pm}$) in ILP model (1) can be whitened as any real value within its boundaries. For any combination of whitened values of interval-set coefficients (abbreviated as whitened coefficients), there might be an optimal solution that can optimize objective function (1-1) subject to whitened constraints. Infiniteness of parameter combinations leads to that of optimal solutions. All optimal solutions constitute a decision space which is denoted as DILPMax. For any solution in DILPMax, it is optimal for at least one combination of whitened coefficients. All decision alternatives for management under interval uncertainties are located in DILPMax. However, DILPMax is an irregular space which is hardly intuitive for decision makers. There are complicated interactive relationships among best solutions of all decision variables. In contrast, a hypercube decision space in which alternatives of any decision variable fluctuate within a range and are independent with that of other decision variables is of higher applicability for engineering practices.

Potential methods are to contract or extend DILPMax to a hypercube space. Let the hypercube space obtained from two operations be denoted as DILPHL and DILPHU, respectively. We have DILPHL $\subseteq$ DILPMax and DILPMax $\subseteq$ DILPHU. Let the difference between decision spaces DILPMax and DILPHU be denoted as DILPMU. For any solution in DILPMU, there does not exist a combination of whitened coefficients for which it is the optimal solution. Equivalently, it may be a conservative solution that correspond to over-satisfaction of constraints and waste of optimality. It may also be a radical solution that implies violation of constraints and potentials of penalties. Local optimality is disabled for solutions in DILPMU. They should be excluded from the final decision space. In comparison, any solution in decision space DILPHL remains being optimal for at least one combination of whitened coefficients. Contraction of DILPMax to a hypercube space is more desired for real-world REM problems.

As the first attempt to obtain a hypercube decision space for ILP problems through contraction of DILPMax, Huang et al. (1992) developed a two-step approach (TSA). Prior to presentation of formulations of TSA in the next sub-section, multiple assumptions on which reliability of the TSA approach relies and which are inherent with most real-world ILP systems are summarized as follows. These assumptions are also required for the proposed IRLP approach.

Assumption 1: Zero is not included in all interval coefficients such as $\left\{c_{j}^{ \pm} \mid j=1,2, \ldots, n\right\},\left\{a_{i j}{ }^{ \pm} \mid i=1,2, \ldots, m ; j=1\right.$, $2, \ldots, n\}$ and $\left\{b_{i}^{ \pm} \mid i=1,2, \ldots, m\right\}$ unless it is the lower or upper bound. Namely, the product of bounds of any interval coefficient is not less than zero.

Assumption 2: Values of any decision variable are nonegative. It holds for all interval sets that the lower bound is not greater than the upper bound. Thus, we can have both the lower bound and the upper bound of interval-valued decision variable $x_{j}^{ \pm}(j=1,2, \ldots, n)$ are not less than zero as stated by constraints (1-3).

Assumption 3: Let the set of all interval coefficients in $I L P$ model (1) be denoted as $\boldsymbol{I C}$, i.e., $\boldsymbol{I C}=\left\{c_{j} \mid c_{j}^{-} \leq c_{j} \leq c_{j}^{+} ; j=\right.$ $1,2, \ldots, n\} \cup\left\{a_{i j} \mid a_{i j}{ }^{-} \leq a_{i j} \leq a_{i j}{ }^{+} ; i=1,2, \ldots, m ; j=1,2, \ldots\right.$, $n\} \cup\left\{b_{i} \mid b_{i}{ }^{-} \leq b_{i} \leq b_{i}{ }^{+} ; i=1,2, \ldots, m\right\}$. For any two different elements in $\boldsymbol{I C}$, they would fluctuate within corresponding interval bounds independently.

Assumption 4: There is at least one optimal solution for any combination of all coefficients in ILP model (1) as the fluctuation of coefficients within interval bounds. This is equivalent to the existence of at least one feasible solution for any combination of coefficients $\left\{a_{i j}^{ \pm} \mid i=1,2, \ldots, m ; j=1,2, \ldots\right.$, $n\}$ and $\left\{b_{i}{ }^{ \pm} \mid i=1,2, \ldots, m\right\}$ in constraints (1-2) subject to constraints (1-3).

Assumption 5: It holds for interval coefficients in the objective function (1-1) that $c_{j}^{ \pm} \geq 0$ (for $j=1,2, \ldots, k$ ) and $c_{j}^{ \pm}<0$ (for $j=k+1, k+2, \ldots, n$ ) where $k \in\{1,2, \ldots, n\}$. This could be achieved by reordering decision variables and corresponddingly coefficients if this assumption cannot be directly satisfied.

\subsection{Initialization of Decision Space}

Based on analysis of interactions among decision variables, coefficients and the objective function under interval uncertainties, two interactive $L P$ sub-models, as an equivalence of the initial ILP model, are constructed on the basis of assumptions 1 to 5. The first sub-model (denoted as ILPI) can be formulated as:

$\max F^{+}=\sum_{j \in J_{1}}\left(c_{j}^{+} \cdot x_{j}^{+}\right)+\sum_{j \in J_{2}}\left(c_{j}^{+} \cdot x_{j}^{-}\right)$

subject to

$\sum_{j \in J_{1}}\left(\operatorname{sign}\left(a_{i j}^{ \pm}\right) \cdot\left|a_{i j}\right|^{-} \cdot x_{j}^{+}\right)$

$+\sum_{j \in J_{2}}\left(\operatorname{sigh}\left(a_{i j}^{ \pm}\right) \cdot\left|a_{i j}^{ \pm}\right|^{+} \cdot x_{j}^{-}\right)$

$\leq b_{i}^{+}$for any $i \in \boldsymbol{I}$

$x_{j}^{+} \geq 0$ for any $j \in J_{1}$

$x_{j}^{-} \geq 0$ for any $j \in J_{2}$

where symbols ${ }^{-}$and ${ }^{+}$denote the lower and upper bounds of interval sets, respectively; $\boldsymbol{I}=\{1,2, \ldots, m\}, \boldsymbol{J}_{1}=\{1,2, \ldots, k\}$, 
$\boldsymbol{J}_{2}=\{k+1, k+2, \ldots, n\}$, and $\boldsymbol{J}=\{1,2, \ldots, n\} ;$ sign function $\operatorname{sign}\left(a_{i j}{ }^{ \pm}\right)$equals to 1 if and only if $a_{i j}{ }^{+} \geq a_{i j}{ }^{-} \geq 0$, and equals to -1 if and only if $a_{i j}{ }^{-}<a_{i j}{ }^{+}<0 ;\left|a_{i j}{ }^{ \pm}\right|$is the absolute value of interval coefficient $a_{i j}{ }^{ \pm}(i \in \boldsymbol{I} ; j \in \boldsymbol{J}) ;\left|a_{i j}{ }^{ \pm}\right|=\left[a_{i j}{ }^{-}, a_{i j}{ }^{+}\right]$if $a_{i j}{ }^{+} \geq$ $a_{i j}{ }^{-} \geq 0$, and $\left|a_{i j}{ }^{ \pm}\right|=\left[-1 \cdot a_{i j}{ }^{+},-1 \cdot a_{i j}{ }^{-}\right]$if $0 \geq a_{i j}{ }^{+} \geq a_{i j}{ }^{-}$; decision variables are upper bounds of the first $k$ interval decision variables in ILP model (1), i.e., $x_{j}^{+}\left(j \in J_{1}\right)$, as well as lower bounds of the latter $(n-k)$ ones, i.e., $x_{j}^{-}\left(j \in J_{2}\right)$.

Model ILPI is a crisp linear programming model. All coefficients in either the objective function (2-1) or constraints (22) are constants instead of interval sets. The simplex method (Dantzig, 1963), as an efficient algorithm for linear programmming problems, is employed to solve $L P$ model $I L P 1$. Let the optimum values of decision variables be denoted as $x_{\text {opt }}{ }^{+}(j \in$ $\left.\boldsymbol{J}_{1}\right)$ and $x_{\text {jopt }}{ }^{-}\left(j \in \boldsymbol{J}_{2}\right)$. The corresponding objective function value (denoted as ${F_{\text {opt }}}^{+}$) can be obtained by ${F_{\text {opt }}}^{+}=\sum_{j \in J_{1}}\left(c_{j}{ }^{+} \cdot x_{j o p t}{ }^{+}\right)$ $+\sum_{j \in J_{2}}\left(c_{j}^{+} \cdot x_{\text {jopt }}{ }^{-}\right)$. Solutions of model ILPI are preferred by optimistic decision makers who pursue higher system optimality while taking higher constraint-violation risks. Based on solutions $x_{\text {jopt }}{ }^{+}\left(j \in J_{1}\right)$ and $x_{j o p t}{ }^{-}\left(j \in J_{2}\right)$, the second sub-model (denoted as $I L P 2)$ can be formulated as:

$\max F^{-}=\sum_{j \in J_{1}}\left(c_{j}^{-} \cdot x_{j}^{-}\right)+\sum_{j \in J_{2}}\left(c_{j}^{-} \cdot x_{j}^{+}\right)$

subject to

$\sum_{j \in J_{1}}\left(\operatorname{sign}\left(a_{i j}^{ \pm}\right) \cdot \mid a_{i j}{ }^{ \pm+} \cdot x_{j}^{-}\right)$

$+\sum_{j \in J_{2}}\left(\operatorname{sign}\left(a_{i j}^{ \pm}\right) \cdot\left|a_{i j}\right|^{-} \cdot x_{j}^{+}\right)$

$\leq b_{i}^{-}$for any $i \in \boldsymbol{I}$

$x_{\text {opt }}{ }^{+} \geq x_{j}^{-} \geq 0$ for any $j \in J_{1}$

$x_{j}^{+} \geq x_{\text {jopt }}^{-}$for any $j \in \boldsymbol{J}_{2}$

where symbols ${ }^{-}$and ${ }^{+}$, sign functions $\operatorname{sign}\left(a_{i j}{ }^{ \pm}\right)$, and absolute values of interval coefficients (i.e., $\left|a_{i j}{ }^{ \pm}\right|$) are defined as that in model $I L P I$; decision variables are lower bounds of the first $k$ interval decision variables in $I L P$ model (1), i.e., $x_{j}^{-}\left(j \in \boldsymbol{J}_{1}\right)$, as well as upper bounds of the latter $n-k$ ones, i.e., $x_{j}^{+}\left(j \in J_{2}\right)$.

The connection between models ILPI and ILP2 is reflected by constraints (3-3) and (3-4). For any interval-valued decision variable $x_{j}^{ \pm}(j \in \boldsymbol{J})$, the lower bound $\left(x_{j}^{-}\right)$cannot be greater than the upper one $\left(x_{j}^{+}\right)$although they are obtained from two separate models. This connection is necessary for guaranteeing continuity of the initial decision space. Model ILP2 is also a deterministic linear programming model without interval coefficients. The simplex method (Dantzig, 1963) is employed again for obtaining the optimum values of decision variables. Let them be denoted as $x_{j o p t}{ }^{-}\left(j \in \boldsymbol{J}_{1}\right)$ and $x_{\text {jopt }}{ }^{+}\left(j \in \boldsymbol{J}_{2}\right)$. Let the resulting objective function value be denoted as $F_{\mathrm{opt}}{ }^{-}$. It equals to $\sum_{j \in J_{1}}\left(c_{j}^{-} \cdot x_{j o p t}{ }^{-}\right)+\sum_{j \in J_{2}}\left(c_{j}^{-} \cdot x_{j \mathrm{opt}}{ }^{+}\right)$. These solutions represent lower system optimality and lower constraint-violation risks, thus they are pursued by conservative decision makers.

Coupling optimal solutions of sub-models ILPI and ILP2 leads to the optimum interval-set solution (denoted as $x_{\text {jopt }}{ }^{ \pm}$where $j \in J$ ) of decision variables. For any $j \in J$, we can have $x_{\text {jopt }}{ }^{ \pm}$ $=\left[x_{\text {jopt }}{ }^{-}, x_{\text {jopt }}{ }^{+}\right]$where $x_{\text {jopt }}{ }^{-}$and $x_{\text {jopt }}{ }^{+}$are obtained from interacttive $L P$ models ILPI and ILP2. For example, $x_{1 \text { opt }}{ }^{ \pm}=\left[x_{1 \mathrm{opt}}{ }^{-}\right.$, $\left.x_{1 \text { opt }}{ }^{+}\right]$where $x_{1 \text { opt }}{ }^{-}$and $x_{\text {lopt }}{ }^{+}$are obtained from ILP2 and ILPI, respectively. For ILP model (1), the initial decision space obtained from models $I L P 1$ and $I L P 2$ is $\left\{\left(x_{1}, x_{2}, \ldots, x_{n}\right) \mid x_{j} \in\left[x_{j o p t}{ }^{-}\right.\right.$, $\left.x_{j \mathrm{opt}}{ }^{+}\right]$for $\left.j \in \boldsymbol{J}\right\}$. Let it be denoted as $I D S$.

\subsection{Significance of ILP}

Due to high reliability on providing desired decision schemes for ILP problems, the ILP model and the TSA approach have been extended to planning of a large number of resources and environmental programming problems under interval uncertainties and other system complexities. Among them, representative ones included, but not limited to, the followings:

(a) Waste management (Huang et al., 1992; Chang et al., 1997; Maqsood and Huang, 2003; Huang et al., 2005a; Huang et al., 2005b; Pires et al., 2011; Sun et al., 2013; Wu et al, 2015; $\mathrm{Xu}$ et al., 2014),

(b) Water resources allocation (Chang et al., 1997; Wu et al., 1997; Huang and Loucks, 2000; Maqsood et al., 2005; Li et al., 2008; Lv et al., 2010; Zhang et al., 2011; Nikoo et al., 2012; Miao et al., 2014),

(c) Energy systems management (Lin et al., 2005; Cai et al., 2009; Dong et al., 2012; Li et al., 2014; Hu et al., 2014),

(d) Air quality control (Huang, 1992; Liu et al., 2003; Li et al., 2006; Lu et al., 2008; Qin et al., 2010; Wang and Huang, 2013; Shao et al., 2014),

(e) Flood mitigation (Xia et al., 2001),

(f) Water quality management (Huang, 1996; Zeng et al., 2000; Guo et al., 2003; Jin et al., 2004; Qin et al., 2009; Nikoo et al., 2012; Hu et al., 2013; Liu et al., 2015),

( $g$ ) Eutrophication control (Huang et al., 2012),

(h) Land-use planning (Huang et al., 1998; Han et al., 2013; Xu et al., 2014; Ni et al., 2014; Zhang et al., 2014),

(i) Resources management (Xia et al., 2001; Nasiri and Huang, 2007; Li et al., 2011; You et al., 2014; Wang et al., 2014; Niu et al., 2014),

(j) Carbon dioxide trading planning (Li et al., 2011; Chen et al., 2013; Zhu et al., 2015), and

(k) Transportation planning (Nasiri et al., 2009; Yan et al., 2010; Tan et al., 2011).

The initial decision space (IDS) is an $n$-dimension hypercube. It is supposed that there is at least one combination of whitened coefficients, i.e., an element $\{\boldsymbol{C}, \boldsymbol{A}, \boldsymbol{b}\}$ of set $\left\{\left\{\left\{c_{j}\right\}^{1 \times n}\right.\right.$, $\left.\left\{a_{i j}\right\}^{m \times n},\left\{b_{i}\right\}^{m \times 1}\right\} \mid c_{j}^{-} \leq c_{j} \leq c_{j}^{+}$for $j \in \boldsymbol{J} ; a_{i j} \leq a_{i j} \leq a_{i j}{ }^{+}$for $i \in \boldsymbol{I}$ and $j \in \boldsymbol{J} ; b_{i}^{-} \leq b_{i} \leq b_{i}^{+}$for $\left.i \in \boldsymbol{I}\right\}$, such that $\boldsymbol{X}_{0}$ is the optimal solution for $\max \{\boldsymbol{C X} \mid \boldsymbol{A} \boldsymbol{X} \leq \boldsymbol{b} ; \boldsymbol{X} \geq \boldsymbol{0}\}$ where $\boldsymbol{X}_{0}$ is any combination of decision variables belonging to IDS. This can be defined as continuity of the initial decision space. In comparison with discrete interval linear programming (DILP) methods (e.g., robust linear programming) where only one or two discrete decision schemes could be obtained, more alternatives can be provided by the initial continuous decision space for deci- 
sion making under interval uncertainties. One-sidedness of a single decision scheme in DILP can be avoided through introduction of interval-set decision variables and proposition of the TSA approach. This is helpful for increasing reliabilities of ILP methods for real-world ILP problems in which decision makers are diversified in preferences. In addition, evaluation of application efficiencies indicates that the ILP model is capable of effectively reflecting interval uncertainties in ILP problems, incorporating them into the optimization process, and enhancing robustness of related modeling efforts.

\section{Violation Analysis}

\subsection{Violation Criterion and Ranges}

As stated above, two optimal solutions are obtained from the simplex method on two correlated $L P$ sub-models. The initial decision space (i.e., $I D S$ ) is constructed by coupling them together. In addition to the two solutions, infinite solutions are included in IDS. It is possible that constraints of either sub-model are violated by solutions in IDS, of which the occurrence likeliness varies with sub-models ILPI and ILP2. Sub-model $I L P 1$ aims to obtain an optimistic solution of ILP model (1) under continuity of the initial decision space and intervalness of system properties. The solution is preferred by decision makers who pursue high system profits under high risks of constraint violation. In contrast, sub-model ILP2 is developed for identifying a scheme desired for conservative decision makers who prefer to mitigate constraint violation risks at the cost of high system profits. A few of solutions in IDS may violate constraints of sub-model $I L P I$, which may lead to high penalties because of optimism of ILP1. As for sub-model ILP2, constraint violation may hold for more solutions in $I D S$, and the average penalty is lower than sub-model ILPI due to conservativeness of sub-model $I L P 2$. High penalties from constraint violation of submodel ILPI are usually unaffordable in real-world management problems under interval uncertainties. Therefore, the criterion of constraint violation of ILP model (1) is identified as violation of constraints of sub-model ILPI in this study. The criterion can be others such as violation of constraints that correspond to the highest system optimality or security, which will be examined in future studies.

Assumption 6: The criterion of constraint violation of ILP model (1) is violation of constraints of sub-model ILPI for all solutions in the initial decision space.

Let the left-hand side of sub-model ILPI (2) be abbreviated as $\left(\boldsymbol{A}_{i}{ }^{ \pm} \boldsymbol{X}^{ \pm}\right)_{\text {C }}$, i.e., $\left(\boldsymbol{A}_{i}{ }^{ \pm} \boldsymbol{X}^{ \pm}\right)_{\mathrm{C}}=\sum_{j \in J_{i}}\left(\operatorname{sign}\left(a_{i j}{ }^{ \pm}\right) \cdot\left|a_{i j}{ }^{ \pm}\right|^{-} \cdot x_{j}\right)+\sum_{j \in J_{2}}$ $\left(\operatorname{sign}\left(a_{i j}{ }^{ \pm}\right) \cdot\left|a_{i j}\right|^{+} \cdot x_{j}\right)$ for any $i \in \boldsymbol{I}$. Inequalities (2-2) are equivalent to $\left(\boldsymbol{A}_{i}{ }^{ \pm} \boldsymbol{X}^{ \pm}\right)_{\mathrm{C}} \leq b_{i}{ }^{+}$for any. Let $\operatorname{SIGp}()$ and $\operatorname{SIGn}()$ be sign functions of coefficients $a_{i j}^{ \pm}(i \in \boldsymbol{I}$ and $j \in \boldsymbol{J})$. For any $i \in \boldsymbol{I}$ and $j \in$ $J, \operatorname{SIG} p\left(a_{i j}^{ \pm}\right)=1$ and $\operatorname{SIGn}\left(a_{i j}{ }^{ \pm}\right)=0$ if ${a_{i j}}^{ \pm} \geq 0$, and $\operatorname{SIGp}\left(a_{i j}^{ \pm}\right)=$ 0 and $\operatorname{SIGn}\left(a_{i j}^{ \pm}\right)=-1$ if $a_{i j}^{ \pm}<0$. Inequalities (2-2) are equivalent with $\sum_{j \in J_{i}}\left(\operatorname{SIGp}\left(a_{i j}{ }^{ \pm}\right) \cdot\left|a_{i j}{ }^{ \pm}\right|^{-} \cdot x_{j}^{+}\right)+\sum_{j \in J_{1}}\left(\operatorname{SIGn}\left(a_{i j}{ }^{ \pm}\right) \cdot\left|a_{i j}{ }^{ \pm}\right|^{-} \cdot x_{j}^{+}\right)+$ $\sum_{j \in J_{2}}\left(\operatorname{SIGp}\left(a_{i j}^{ \pm}\right) \cdot\left|a_{i j}\right|^{+} \cdot x_{j}^{-}\right)+\sum_{j \in J_{2}}\left(\operatorname{SIGn}\left(a_{i j}{ }^{ \pm}\right) \cdot\left|a_{i j}{ }^{ \pm}\right|^{+} \cdot x_{j}^{-}\right) \leq b_{i}^{+}$for any $i \in I$. With fluctuation of decision variables within initial decision space $I D S$, i.e., $\left\{\left(x_{1}, x_{2}, \ldots, x_{n}\right) \mid x_{j} \in\left[x_{\text {jopt }}{ }^{-}, x_{j \mathrm{opt}}{ }^{+}\right]\right.$for $j$ $\in \boldsymbol{J}\}$, the left-hand side of inequalities (2-2) can be maximized at $\sum_{j \in J_{1}}\left(\operatorname{SIGp}\left(a_{i j}{ }^{ \pm}\right) \cdot\left|a_{i j}\right|^{ \pm} \cdot x_{j \mathrm{opt}}{ }^{+}\right)+\sum_{j \in J_{1}}\left(\operatorname{SIGn}\left(a_{i j^{ \pm}}{ }^{ \pm}\right) \cdot\left|a_{i j^{ \pm}}\right|^{-} \cdot x_{j \mathrm{opt}}{ }^{-}\right)+$ $\sum_{j \in J_{2}}\left(\operatorname{SIGp}\left(a_{i j}{ }^{ \pm}\right) \cdot\left|a_{i j}\right|^{ \pm+} \cdot x_{j \mathrm{opt}}{ }^{+}\right)+\sum_{j \in J_{2}}\left(\operatorname{SIGn}\left(a_{i j}{ }^{ \pm}\right) \cdot\left|a_{i j}\right|^{{ }^{+} \cdot} x_{j \mathrm{opt}}{ }^{-}\right)$where $i \in I$. Namely, Max $\left\{\sum_{j \in J_{1}}\left(\operatorname{SIG} p\left(a_{i j}{ }^{ \pm}\right) \cdot\left|a_{i j}{ }^{ \pm}\right|^{-} \cdot x_{j \mathrm{opt}}\right)+\sum_{j \in J_{1}}(S I G n\right.$ $\left.\left(a_{i j}{ }^{ \pm}\right) \cdot\left|a_{i j}{ }^{ \pm}\right|^{-} \cdot x_{j \mathrm{opt}}\right)+\sum_{j \in J_{2}}\left(\operatorname{SIGp}\left(a_{i j}{ }^{ \pm}\right) \cdot\left|a_{i j}{ }^{ \pm}\right|^{+} \cdot x_{j \mathrm{opt}}\right)+\sum_{j \in J_{2}}\left(\operatorname{SIGn}\left(a_{i j}{ }^{ \pm}\right)\right.$ $\left.\left.\cdot\left|a_{i j}\right|^{+} \cdot x_{j \mathrm{opt}}\right) \mid\left(x_{1}, x_{2}, \ldots, x_{n}\right) \in I D S\right\}=\sum_{j \in J_{1}}\left(\operatorname{SIGp}\left(a_{i j}{ }^{ \pm}\right) \cdot\left|a_{i j}\right|^{ \pm} \cdot x_{j \mathrm{opt}}{ }^{+}\right)$ $+\sum_{j \in J_{1}}\left(\operatorname{SIGn}\left(a_{i j}{ }^{ \pm}\right) \cdot\left|a_{i j}{ }^{ \pm}\right|^{-} \cdot x_{j \mathrm{opt}}{ }^{-}\right)+\sum_{j \in J_{2}}\left(\operatorname{SIGp}\left(a_{i j}{ }^{ \pm}\right) \cdot\left|a_{i j}{ }^{ \pm}\right|^{+} \cdot x_{j \mathrm{opt}}{ }^{+}\right)+$ $\sum_{j \in J_{2}}\left(\operatorname{SIGn}\left(a_{i j}{ }^{ \pm}\right) \cdot\left|a_{i j}\right|^{+} \cdot x_{j \text { opt }}{ }^{-}\right)$for any $i \in I$. Therefore, violation of the $i$ th $(i \in I)$ constraint of model ILPI is equivalent with $\sum$ ${ }_{j \in J_{1}}\left(\left.\operatorname{SIGp}\left(a_{i j}{ }^{ \pm}\right) \cdot\left|a_{i j}\right|^{ \pm}\right|^{-} \cdot x_{j \mathrm{opt}}{ }^{+}\right)+\sum_{j \in J_{1}}\left(\left.\operatorname{SIGn}\left(a_{i j}{ }^{ \pm}\right) \cdot\left|a_{i j}\right|^{ \pm}\right|^{-} \cdot x_{j \mathrm{opt}}{ }^{-}\right)+\sum_{j \in J_{2}}$ $\left(\operatorname{SIGp}\left(a_{i j}{ }^{ \pm}\right) \cdot\left|a_{i j}\right|^{+} \cdot x_{j \mathrm{opt}}{ }^{+}\right)+\sum_{j \in J_{2}}\left(\operatorname{SIGn}\left(a_{i j}{ }^{ \pm}\right) \cdot \mid a_{i j}{ }^{ \pm+} \cdot x_{j \mathrm{opt}}{ }^{-}\right)>b_{i}^{+}$.

Let violated constraints be denoted as a set $\boldsymbol{I} \boldsymbol{V}=\{i(1), i(2)$, $\ldots, i(V)\}$ where $V \in \boldsymbol{V}$ and $\boldsymbol{V}=\{1,2, \ldots, m\}$. For any $v \in \boldsymbol{V}$, the $i(v)$ th constraint in the first sub-model (2) is violated. Equivalently, we have $\sum_{j \in J_{1}}\left(\operatorname{SIGp}\left(a_{i(v) j_{j}}\right)^{ \pm} \cdot\left|a_{i(v) j}\right|^{ \pm} \mid \cdot x_{j \mathrm{opt}}{ }^{+}\right)+\sum_{j \in J_{1}}(S I G n$ $\left.\left(a_{i(v) j}{ }^{ \pm}\right) \cdot\left|a_{i(v) j} j^{ \pm}\right|^{-} \cdot x_{j \mathrm{opt}}{ }^{-}\right)+\sum_{j \in J_{2}}\left(\left.\operatorname{SIGp}\left(a_{i(v) j^{ \pm}}\right) \cdot\left|a_{i(v) j}\right|^{+}\right|^{+} \cdot x_{j \mathrm{opt}}{ }^{+}\right)+\sum_{j \in J_{2}}$ $\left(\operatorname{SIGn}\left(a_{i(v) j}{ }^{ \pm}\right) \cdot\left|a_{i(v) j}\right|^{ \pm} \cdot x_{j \mathrm{opt}}{ }^{-}\right)>b_{i(v)}{ }^{+}$for any $v \in \boldsymbol{V}$.

Definition 1: The violation range of the $i(v)$ th constraint, denoted as $V R_{i(v)}$, is the range of the right-hand coefficient (i.e., $\left.b_{i(v)}{ }^{+}\right)$being violated by the maximum value of $\left(\boldsymbol{A}_{i}{ }^{ \pm} \boldsymbol{X}^{ \pm}\right)_{\mathrm{C}}$. It equa1s to $V R_{i(v)}=\sum_{j \in J_{1}}\left(\operatorname{SIGp}\left(a_{i(v) j^{ \pm}}\right) \cdot \mid a_{i(v) j} j^{ \pm} \cdot x_{j o p t}{ }^{+}\right)+\sum_{j \in J_{1}}\left(\operatorname{SIGn}\left(a_{i(v) j}{ }^{ \pm}\right)\right.$ $\left.\cdot\left|a_{i(v) j}{ }^{ \pm}\right|^{-} \cdot x_{j \mathrm{opt}}{ }^{-}\right)+\sum_{j \in J_{2}}\left(\operatorname{SIGp}\left(a_{i(v) j}{ }^{ \pm}\right) \cdot\left|a_{i(v) j}{ }^{ \pm}\right|^{+} \cdot x_{j \mathrm{opt}}{ }^{+}\right)+\sum_{j \in J_{2}}(S I G n$ $\left.\left(a_{i(v) j}^{ \pm}\right) \cdot\left|a_{i(v) j}\right|^{+} \cdot x_{\text {opt }}{ }^{-}\right)-b_{i(v)}{ }^{+}$for any $v \in \boldsymbol{V}$.

\subsection{Contraction Ranges}

To mitigate the problem of constraint violation in the initial decision space, violation range $V R_{i(v)}$ should be contracted as zero for any $v \in \boldsymbol{V}$. This can be achieved through contraction of four groups of decision variables, i.e.: i) the upper bound of the first $k$ interval decision variables with non-negative technical coefficients (i.e., $x_{\text {jopt }}{ }^{+}$where $j \in J_{1}$ and $a_{i(v) j}{ }^{ \pm} \geq 0$ ); ii) the lower bound of the first $k$ interval decision variables with negative technical coefficients (i.e., $x_{\text {jopt }}{ }^{-}$where $j \in J_{1}$ and $a_{i(v) j}{ }^{ \pm}<0$ ); iii) the upper bound of the last $n-k$ interval decision variables with non-negative technical coefficients (i.e., $x_{\text {jopt }}{ }^{+}$where $j \in J_{2}$ and $a_{i(v) j}{ }^{ \pm} \geq 0$ ); and iv) the lower bound of the last $n-k$ interval decision variables with negative coefficients (i.e., $x_{j \text { opt }}{ }^{-}$where $j$ $\in J_{2}$ and $\left.a_{i(v) j}{ }^{ \pm}<0\right)$. For the convenience of expression in the followings, the first and fourth groups of decision variables are denoted as type-II decision variables, and the other two groups as type-I decision variables.

Let solutions after contraction be denoted as $x_{\text {jopt* }}{ }^{ \pm}(j \in J)$. The $i(v)$ th $(v \in V)$ constraint in model ILPI is not violated if and only if $\sum_{j \in J_{1}}\left(\operatorname{SIGp}\left(a_{i(v) j}{ }^{ \pm}\right) \cdot\left|a_{i(v) j}\right|^{ \pm} \cdot x_{j o \mathrm{opt}^{*}}{ }^{+}\right)+\sum_{j \in J_{1}}\left(\operatorname{SIGn}\left(a_{i(v) j} j^{ \pm}\right)\right.$

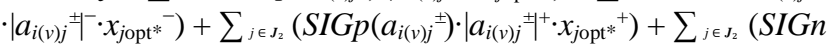
$\left.\left.\left(a_{i(v) j}{ }^{ \pm}\right) \cdot\left|a_{i(v) j}\right|^{+} \cdot x_{j \mathrm{opt}^{*}}\right) \leq b_{i(v)}\right)^{+}$. Therefore, the overall contraction range of the second and third groups of decision variables in $\left(\boldsymbol{A}_{i(v)}{ }^{ \pm} \boldsymbol{X}^{ \pm}\right)_{\mathrm{C}}$, denoted as $S R_{i(v) 1}$, equals to $\sum_{j \in J_{1}}\left(\operatorname{SIGn}\left(a_{i(v) j}{ }^{ \pm}\right) \cdot\left|a_{i(v) j}\right|^{ \pm}\right.$ $\left.\cdot\left(x_{j o p t}{ }^{-}-x_{j o p t^{*}}\right)\right)+\sum_{j \in J_{2}}\left(\left.\operatorname{SIGp}\left(a_{i(v) j}{ }^{ \pm}\right) \cdot\left|a_{i(v) j}\right|^{+}\right|^{+} \cdot\left(x_{j o p t}{ }^{+}-x_{j o p t} *^{+}\right)\right)$. The maximum value of $S R_{i(v) 1}$, i.e., the type-I maximum contraction range of $\left(\boldsymbol{A}_{i(v)}{ }^{ \pm} \boldsymbol{X}^{ \pm}\right)_{\mathrm{C}}$ (abbreviated as $\left.M S R_{1 i(v)}\right)$, is $M S R_{1 i(v)}=\sum_{j \in J_{1}}$ $\left(\operatorname{SIGn}\left(a_{i(v) j}{ }^{ \pm}\right) \cdot\left|a_{i(v) j j^{ \pm}}\right|^{-} \cdot\left(x_{j \mathrm{opt}}{ }^{-}-x_{j \mathrm{opt}}{ }^{+}\right)\right)+\sum_{j \in J_{2}}\left(\operatorname{SIGp}\left(a_{i(v) j}{ }^{ \pm}\right) \cdot\left|a_{i(v) j}\right|^{ \pm}\right.$ $\left.\cdot\left(x_{j \text { opt }}{ }^{+}-x_{j \text { opt }}{ }^{-}\right)\right)$. Similarly, the overall contraction range of the first and fourth groups of decision variables in $\left(\boldsymbol{A}_{i(v)}{ }^{ \pm} \boldsymbol{X}^{ \pm}\right)_{\mathrm{C}}$, deno- 
ted as $S R_{i(v) 2}$, equals to $\sum_{j \in J_{1}}\left(\operatorname{SIGp}\left(a_{i(v) j^{ \pm}}\right) \cdot\left|a_{i(v) j}\right|^{ \pm} \cdot\left(x_{j \mathrm{opt}}{ }^{+}-x_{j \mathrm{opt}^{*}}{ }^{+}\right)\right)$ $+\sum_{j \in J_{2}}\left(\operatorname{SIGn}\left(a_{i(v) j}{ }^{ \pm}\right) \cdot\left|a_{i(v) j}\right|^{+} \cdot\left(x_{j \mathrm{opt}}{ }^{-}-x_{j \mathrm{opt} *^{-}}\right)\right)$. Its maximum value is $\sum_{j \in J_{1}}\left(\operatorname{SIGp}\left(a_{i(v) j}{ }^{ \pm}\right) \cdot\left|a_{i(v) j^{ \pm}}\right|^{-} \cdot\left(x_{j \mathrm{opt}}{ }^{+}-x_{\text {jopt }}{ }^{-}\right)\right)+\sum_{j \in J_{2}}\left(\operatorname{SIGn}\left(a_{i(v) j^{ \pm}}\right)\right.$. $\left.\left|a_{i(v) j}\right|^{ \pm} \cdot\left(x_{\text {jopt }}{ }^{-}-x_{\text {jopt }}{ }^{+}\right)\right)$, which is named as the type-II maximum contraction range of $\left(\mathbf{A}_{i(v)}{ }^{ \pm} \boldsymbol{X}^{ \pm}\right)_{\mathrm{C}}$ and abbreviated as $M S R_{2 i(v)}$.

\section{Proposition 1:}

(a) $V R_{i(v)} \leq M S R_{1 i(v)}$ for any $v \in V$;

(b) $V R_{i(v)}=M S R_{1 i(v)}$ if and only if $\left(\boldsymbol{A}_{i(v)}{ }^{ \pm} \boldsymbol{X}^{ \pm}\right)_{\mathrm{C}}=b_{i(v)}{ }^{+}(v \in \boldsymbol{V})$;

(c) $S R_{i(v) 1}+S R_{i(v) 2} \geq V R_{i(v)}$ for any $v \in V$;

(d) $S R_{i(v) 1} \leq M S R_{1 i(v)}$ for any $v \in V$; and

(e) $S R_{i(v) 2} \leq M S R_{2 i(v)}$ for any $v \in V$.

Proof:

(a) For any $v \in V, M_{1 i(v)}-V R_{i(v)}=\left\{\sum_{j \in J_{1}}\left(\operatorname{SIGn}\left(a_{i(v) j^{ \pm}}\right)\right.\right.$. $\left.\left|a_{i(v) j}\right|^{ \pm} \cdot\left(x_{j \mathrm{opt}}{ }^{-}-x_{\text {jopt }}{ }^{+}\right)\right)+\sum_{j \in J_{2}}\left(\operatorname{SIGp}\left(a_{i(v) j}{ }^{ \pm}\right) \cdot\left|a_{i(v) j}{ }^{ \pm}\right|^{+} \cdot\left(x_{\text {jopt }}{ }^{+}-x_{\text {jopt }}{ }^{-}\right)\right.$ )$\}-\left\{\sum_{j \in J_{1}}\left(\operatorname{SIGp}\left(a_{i(v) j}\right)^{ \pm}\right) \cdot\left|a_{i(v) j}\right|^{-} \cdot x_{j \mathrm{opt}}\right)^{+}+\sum_{j \in J_{1}}\left(\operatorname{SIGn}\left(a_{i(v) j}{ }^{ \pm}\right) \cdot\left|a_{i(v) j}{ }^{ \pm}\right|\right.$ $\left.\cdot x_{j o p t}{ }^{-}\right)+\sum_{j \in J_{2}}\left(\operatorname{SIGp}\left(a_{i(v) j}{ }^{ \pm}\right) \cdot\left|a_{i(v) j j^{ \pm}}\right|^{+} \cdot x_{j \mathrm{opt}}{ }^{+}\right)+\sum_{j \in J_{2}}\left(\operatorname{SIGn}\left(a_{i(v) j^{ \pm}}\right) \cdot\right.$ $\left.\left.\left|a_{i(v) j}\right|^{+} \cdot x_{j \text { opt }}{ }^{-}\right)-b_{i(v)}{ }^{+}\right\}$that can be simplified as $b_{i(v)}{ }^{+}-\sum_{j \in J_{1}}(S I G p$ $\left.\left(a_{i(v) j}{ }^{ \pm}\right) \cdot\left|a_{i(v) j}\right|^{ \pm} \cdot x_{j \mathrm{opt}}{ }^{+}\right)-\sum_{j \in J_{1}}\left(\operatorname{SIGn}\left(a_{i(v) j} j^{ \pm}\right) \cdot\left|a_{i(v) j}\right|^{ \pm} \cdot x_{j \mathrm{opt}}{ }^{+}\right)-\sum_{j \in J_{2}}$ $\left(\operatorname{SIGp}\left(a_{i(v) j j^{ \pm}}\right) \cdot\left|a_{i(v) j j^{ \pm}}\right|^{+} \cdot x_{j \mathrm{opt}}{ }^{-}\right)-\sum_{j \in J_{2}}\left(\left.\operatorname{SIGn}\left(a_{i(v) j j^{ \pm}}\right) \cdot\left|a_{i(v) j}\right|^{ \pm}\right|^{+} \cdot x_{j \mathrm{opt}}{ }^{-}\right)$. Since the $i(v)$ th constraint is violated for initialized solutions, we have $\sum_{j \in J_{1}}\left(\operatorname{SIGp}\left(a_{i(v) j}{ }^{ \pm}\right) \cdot\left|a_{i(v) j^{ \pm}}\right|^{-} \cdot x_{j \mathrm{opt}^{+}}{ }^{+}\right)+\sum_{j \in J_{1}}\left(\operatorname{SIGn}\left(a_{i(v) j} j^{ \pm}\right)\right.$ $\left.\left|a_{i(v) j}\right|^{ \pm} \cdot x_{j \mathrm{opt}}{ }^{+}\right)+\sum_{j \in J_{2}}\left(\operatorname{SIGp}\left(a_{i(v) j}{ }^{ \pm}\right) \cdot\left|a_{i(v) j}\right|^{ \pm} \cdot x_{j \mathrm{opt}}{ }^{-}\right)+\sum_{j \in J_{2}}(S I G n$ $\left.\left(a_{i(v) j}{ }^{ \pm}\right) \cdot\left|a_{i(v) j}\right|^{+} \cdot x_{\text {jopt }}{ }^{-}\right) \leq b_{i(v)}{ }^{+}$. Equivalently, $V R_{i(v)} \leq M S R_{1 i(v)}$ for any $v \in \boldsymbol{V}$.

(b) For any $v \in \boldsymbol{V}$, it is equivalent with $V R_{i(v)}=M S R_{1 i(v)}$ that $\sum_{j \in J_{1}}\left(\operatorname{SIGp}\left(a_{i(v) j} j^{ \pm}\right) \cdot\left|a_{i(v) j}\right|^{ \pm} \cdot x_{j \mathrm{opt}^{+}}\right)^{+}+\sum_{j \in J_{1}}\left(\operatorname{SIGn}\left(a_{i(v) j_{j}^{ \pm}}\right) \cdot\left|a_{i(v) j}\right|^{ \pm} \cdot\right.$ $\left.x_{\text {jopt }}{ }^{+}\right)+\sum_{j \in J_{2}}\left(\operatorname{SIGp}\left(a_{i(v) j}{ }^{ \pm}\right) \cdot\left|a_{i(v) j}\right|^{+} \cdot x_{j \mathrm{opt}}{ }^{-}\right)+\sum_{j \in J_{2}}\left(\operatorname{SIGn}\left(a_{i(v) j} j^{ \pm}\right) \cdot\right.$ $\left.\left|a_{i(v) j}\right|^{ \pm} \cdot x_{j o p t}{ }^{-}\right)=b_{i(v)}{ }^{+}$. From formulations of $\left(\boldsymbol{A}_{i(v)}{ }^{ \pm} \boldsymbol{X}^{ \pm}\right)_{\mathrm{C}}$, we can have that $\left(\boldsymbol{A}_{i(v)}{ }^{ \pm} \boldsymbol{X}^{ \pm}\right)_{\mathrm{C}}=b_{i(v)}{ }^{+}$.

(c) For any $v \in \boldsymbol{V}, S R_{i(v) 1}+S R_{i(v) 2}-V R_{i(v)}=\left\{\sum_{j \in J_{\mathrm{i}}}(S I G n\right.$ $\left.\left.\left(a_{i(v) j}{ }^{ \pm}\right) \cdot\left|a_{i(v) j}\right|^{ \pm}\right|^{-} \cdot\left(x_{j \mathrm{opt}}{ }^{-}-x_{j \mathrm{opt} \mathrm{*}^{-}}\right)\right)+\sum_{j \in J_{2}}\left(\operatorname{SIGp}\left(a_{i(v) j^{ \pm}}\right) \cdot\left|a_{i(v) j}\right|^{+} \cdot\left(x_{j \mathrm{opt}}{ }^{+}\right.\right.$ $\left.\left.\left.-x_{j o p *^{*}}{ }^{+}\right)\right)\right\}+\left\{\sum_{j \in J_{1}}\left(\operatorname{SIGp}\left(a_{i(v) j}{ }^{ \pm}\right) \cdot\left|a_{i(v) j}\right|^{-} \cdot\left(x_{j o p t}{ }^{+}-x_{j o p t} *^{+}\right)\right)+\sum_{j \in J_{2}}\right.$ $\left.\left(\operatorname{SIGn}\left(a_{i(v) j}{ }^{ \pm}\right) \cdot\left|a_{i(v) j}\right|^{ \pm} \cdot\left(x_{j o p t}{ }^{-}-x_{j o p t^{*}}\right)\right)\right\}-\left\{\sum_{j \in J_{1}}\left(\operatorname{SIGp}\left(a_{i(v) j}{ }^{ \pm}\right) \cdot\right.\right.$ $\left.\left|a_{i(v) j}\right|^{ \pm} \cdot x_{j o p t}{ }^{+}\right)+\sum_{j \in J_{1}}\left(\left.\operatorname{SIGn}\left(a_{i(v) j j^{ \pm}}\right) \cdot\left|a_{i(v) j}\right|^{ \pm}\right|^{-} \cdot x_{j \mathrm{opt}}{ }^{-}\right)+\sum_{j \in J_{2}}(S I G p$ $\left.\left.\left(a_{i(v) j}{ }^{ \pm}\right) \cdot\left|a_{i(v) j}\right|^{+} \cdot x_{\text {jopt }}{ }^{+}\right)+\sum_{j \in J_{2}}\left(\operatorname{SIGn}\left(a_{i(v) j} j^{ \pm}\right) \cdot\left|a_{i(v) j}\right|^{+} \cdot x_{j \mathrm{opt}}{ }^{-}\right)-b_{i(v)}{ }^{+}\right\}$ which is equivalent with $b_{i(v)}{ }^{+}-\left[\sum_{j \in J_{1}}\left(\operatorname{SIGn}\left(a_{i(v) j}\right)^{ \pm}\right)\left|a_{i(v) j j^{ \pm}}\right|^{-}\right.$ $\left.x_{j \mathrm{opt}^{*}{ }^{-}}\right)+\sum_{j \in J_{1}}\left(\operatorname{SIGp}\left(a_{i(v) j}{ }^{ \pm}\right) \cdot\left|a_{i(v) j}\right|^{-} \cdot x_{j \mathrm{opt} *^{+}}\right)+\sum_{j \in J_{2}}\left(\operatorname{SIGp}\left(a_{i(v) j}{ }^{ \pm}\right) \cdot\right.$ $\left.\left.\left.\left|a_{i(v) j}\right|^{ \pm} \cdot x_{j o t^{*}}{ }^{+}\right)+\sum_{j \in J_{2}}\left(\operatorname{SIGn}\left(a_{i(v) j}\right)^{ \pm}\right) \cdot\left|a_{i(v) j}\right|^{+} \cdot x_{j o p t^{*}}\right)\right]$. From the definition of solutions after contraction (i.e., $\left\{x_{\left.\text {jopt* } *^{*^{*}}\right\}^{1 \times n}}\right.$ ), we have $\sum_{j \in J_{1}}\left(\left.\operatorname{SIGp}\left(a_{i(v) j}{ }^{ \pm}\right) \cdot\left|a_{i(v) j}\right|^{ \pm}\right|^{-} \cdot x_{j \mathrm{opt}^{*}}{ }^{+}\right)+\sum_{j \in J_{1}}\left(\operatorname{SIGn}\left(a_{i(v) j}{ }^{ \pm}\right) \cdot\left|a_{i(v) j}{ }^{ \pm}\right|\right.$ $\left.\cdot x_{j o p t^{*}}\right)+\sum_{j \in J_{2}}\left(\operatorname{SIGp}\left(a_{i(v) j}{ }^{ \pm}\right) \cdot\left|a_{i(v) j}{ }^{ \pm}\right|^{+} \cdot x_{j o p t} *^{+}\right)+\sum_{j \in J_{2}}\left(\operatorname{SIGn}\left(a_{i(v) j}{ }^{ \pm}\right)\right.$ $\left.\cdot\left|a_{i(v) j}\right|^{+} \cdot x_{j o p t^{*}}\right) \leq b_{i(v)}{ }^{+}$for any $v \in V$. Thus, $S R_{i(v) 1}+S R_{i(v) 2} \geq V R_{i(v)}$ holds for any $v \in \boldsymbol{V}$.

(d) For any $v \in \boldsymbol{V}, M S R_{1 i(v)}-S R_{i(v) 1}=\left\{\sum_{j \in J_{1}}\left(\operatorname{SIGn}\left(a_{i(v) j^{ \pm}}{ }^{ \pm}\right)\right.\right.$ $\left.\left|a_{i(v) j}\right|^{ \pm} \cdot\left(x_{j o p t}{ }^{-}-x_{j o p t}{ }^{+}\right)\right)+\sum_{j \in J_{2}}\left(\operatorname{SIGp}\left(a_{i(v) j}{ }^{ \pm}\right) \cdot\left|a_{i(v) j}{ }^{ \pm}\right|^{+} \cdot\left(x_{j \mathrm{opt}}{ }^{+}-\right.\right.$ $\left.\left.\left.x_{\text {jopt }}{ }^{-}\right)\right)\right\}-\left\{\sum_{j \in J_{1}}\left(\operatorname{SIGn}\left(a_{i(v) j}{ }^{ \pm}\right) \cdot \mid a_{i(v) j} j^{ \pm} \cdot\left(x_{j \mathrm{opt}}{ }^{-}-x_{j \mathrm{opt}} *^{-}\right)\right)+\sum_{j \in J_{2}}\right.$ $\left.\left.\left(\operatorname{SIGp}\left(a_{i(v) j}\right)^{ \pm}\right) \cdot\left|a_{i(v) j}\right|^{ \pm+} \cdot\left(x_{j \mathrm{opt}}{ }^{+}-x_{j \mathrm{opt}} *^{+}\right)\right)\right\}$which is equivalent with $\sum_{j \in J_{1}}\left(\operatorname{SIGn}\left(a_{i(v) j}{ }^{ \pm}\right) \cdot\left|a_{i(v) j}\right|^{ \pm} \cdot \cdot\left(x_{j o p t t^{-}}-x_{j \mathrm{opt}}{ }^{+}\right)\right)+\sum_{j \in J_{2}}\left(\operatorname{SIGp}\left(a_{i(v) j}{ }^{ \pm}\right) \cdot\right.$ $\left.\left|a_{i(v) j}\right|^{ \pm} \cdot\left(x_{j o t^{*}}{ }^{+}-x_{j o p t}{ }^{-}\right)\right)$. Since $x_{j o p t *}{ }^{-} \in\left[x_{j o p t}{ }^{-}, x_{j o p t}{ }^{+}\right]$as well as $x_{\text {jopt } *^{+}} \in\left[x_{\text {jopt }}{ }^{-}, x_{\text {jopt }}{ }^{+}\right]$for any $j \in J, x_{j o p t t^{-}} \leq x_{\text {jopt }}{ }^{+}$and $x_{j o p t *^{*}} \geq x_{j \text { opt }}{ }^{-}$. Based on definitions of sign functions $\operatorname{SIGp}()$ and $\operatorname{SIGn}()$, we have $\left.\sum_{j \in J_{1}}\left(\operatorname{SIGn}\left(a_{i(v) j}\right)^{ \pm}\right) \cdot\left|a_{i(v) j}\right|^{ \pm} \cdot\left(x_{j \mathrm{opt}}{ }^{-}-x_{j \mathrm{opt}}{ }^{+}\right)\right)+\sum_{j \in J_{2}}(\operatorname{SIG} p$ $\left.\left(a_{i(v) j}{ }^{ \pm}\right) \cdot\left|a_{i(v) j}\right|^{+} \cdot\left(x_{j \mathrm{opt} *^{*}}-x_{\text {jopt }}{ }^{-}\right)\right) \geq 0$. Accordingly, $S R_{i(v) 1} \leq$
$M S R_{1 i(v)}$ for any $v \in \boldsymbol{V}$.

(e) Similarly, inequality $S R_{i(v) 2} \leq M S R_{2 i(v)}$ can be deduced from the relationship between $x_{\text {jopt }}{ }^{ \pm}$and $x_{\text {jopt }}{ }^{ \pm}$and definitions of sign functions.

\subsection{Contraction Ratios}

For any violated constraint $(v \in \boldsymbol{V})$, the overall contraction range of four groups of decision variables, i.e., $S R_{i(v) 1}+S R_{i(v) 2}$, should be greater than or equal to violation range $V R_{i(v)}$. It is implied that the violation problem of any single constraint $(v \in$ $V)$ can be mitigated by infinite alternatives. There is a tradeoff between contraction ranges $S R_{i(v) 1}$ and $S R_{i(v) 2}$ for any violated interval-coefficient constraint. The number of possible contraction schemes for all violated constraints further increases with $V$ exponentially. Therefore, an index named as contraction ratio is proposed to eliminate differences of violation ranges between violated constraints. It is defined as normalization of contraction ranges.

Definition 2: For any $v \in V$, the contraction ratio of typeI decision variables is $S R A_{i(v) 1}=S R_{i(v) 1} / V R_{i(v)}$, while that of $t y$ pe-II decision variables is $S R A_{i(v) 2}=S R_{i(v) 2} / V R_{i(v)}$.

The contraction ratio can be defined as other forms. Defi nition 2 is aimed to intuitively reflect the relationship between parameter values (0/1) and contributions (zero/full) of contraction range for violation range and to avoid complicated submodels. This is helpful for facilitating engineering practices. Besides, the value range of contraction ratios $r_{i(v)}(v \in V)$ is a closed interval $\left[1-M S R_{2 i(v)} / V R_{i(v)}, 1\right]$ if $M S R_{2 i(v)} \leq V R_{i(v)}$; otherwise, it equals to $[0,1]$. According to the definition of contraction ratios, we can have the following corollary.

Corollary 1: If any violated constraint $v \in \boldsymbol{V}$ is satisfied after contraction, then

$$
\begin{aligned}
& \text { (a) } 0 \leq S R A_{i(v) 1} \leq 1 \text { if }\left(\boldsymbol{A}_{i(v)}{ }^{ \pm} \boldsymbol{X}^{ \pm}\right)_{\mathrm{C}}=b_{i(v)}{ }^{+} ; \\
& \text {(b) } 0 \leq S R A_{i(v) 2} \leq M S R_{2 i(v)} / V R_{i(v)} \text {; and } \\
& \text { (c) } S R_{i(v) 2} \geq V R_{i(v)} \cdot(1-r) \text { if } S R A_{i(v) 1} \geq r(r \in[0,1]) .
\end{aligned}
$$

Proof: Straightforward from definitions of parameters such as $S R A_{i(v) 1}, S R A_{i(v) 2}, M S R_{1 i(v)}, M S R_{2 i(v)}, V R_{i(v)}, S R_{i(v) 1}$ and $S R_{i(v) 2}$ and their relationships as stated in proposition 1.

\section{Contraction of Initial Decision Space}

\subsection{Contraction of Optimistic Solutions}

Given a set of minimum contraction ratios $r_{i(v)}$ for violated constraints $(v \in \boldsymbol{V})$, the satisfaction of violated constraints after contraction is equivalent with the following inequalities: $(a)$ $S R A_{i(v) 1} \geq r_{i(v)}$ for any $v \in V,(b) S R A_{i(v) 2} \geq 1-r_{i(v)}$ for any $v \in \boldsymbol{V}$, (c) $x_{j \mathrm{opt} *^{*}} \in x_{j \mathrm{opt}}{ }^{ \pm}$for any $j \in J$, and $(d) x_{j \mathrm{opt} *^{*}} \leq x_{j \mathrm{opt} *^{*}}$ for any $j \in$ $J$. A linear programming sub-model (denoted as ILP3) that is aimed to achieve contraction of type-I decision variables is formulated as:

$\max F_{*^{+}}=\sum_{j \in J_{1}}\left(c_{j}^{+} \cdot x_{j^{*}}\right)+\sum_{j \in J_{2}}\left(c_{j}^{+} \cdot x_{j^{*}}\right)$

subject to 
$S R A_{i(v) 2} \geq 1-r_{i(v)}$ for any $v \in \boldsymbol{V}$

$x_{j^{*}}{ }^{+} \in x_{j \text { opt }}{ }^{ \pm}$for any $j \in \boldsymbol{J}_{1}$

$x_{j^{*}}{ }^{-} \in x_{j o p t}{ }^{ \pm}$for any $j \in \boldsymbol{J}_{2}$

where $x_{j^{*}}{ }^{+}\left(j \in \boldsymbol{J}_{1}\right)$ and $x_{j^{*}}\left(j \in \boldsymbol{J}_{2}\right)$ are decision variables; $S R A_{i(v) 2}(v \in V)$ are contraction ratios for any violated constraint; $x_{j \text { opt }}{ }^{ \pm}(j \in J)$ are interval-set solutions obtained from submodels $I L P I$ and $I L P 2$. Based on formulations of contraction ratios, contraction ranges and maximum contraction ranges, submodel ILP3 can be re-formulated as:

$\max F_{*}^{+}=\sum_{j \in J_{1}}\left(c_{j}^{+} \cdot x_{j^{*}}{ }^{+}\right)+\sum_{j \in J_{2}}\left(c_{j}^{+} \cdot x_{j^{*}}\right)$

subject to

$\sum_{j \in J_{1}}\left(\operatorname{SIGp}\left(a_{i(v) j}{ }^{ \pm}\right) \cdot\left|a_{i(v) j}\right|^{ \pm} \cdot\left(x_{j^{*}}{ }^{+}-x_{j \mathrm{opt}}{ }^{+} \cdot r_{i(v)}\right)\right)$

$+\sum_{j \in J_{1}}\left(\operatorname{SIGn}\left(a_{i(v) j}{ }^{ \pm}\right) \cdot\left|a_{i(v) j}\right|^{-} \cdot x_{j \mathrm{opt}}{ }^{-} \cdot\left(1-r_{i(v)}\right)\right)$

$+\sum_{j \in J_{2}}\left(\operatorname{SIGp}\left(a_{i(v) j} j^{ \pm}\right) \cdot\left|a_{i(v) j}\right|^{+} \cdot x_{j \mathrm{opt}}{ }^{+} \cdot\left(1-r_{i(v)}\right)\right)$

$+\sum_{j \in J_{2}}\left(\operatorname{SIGn}\left(a_{i(v) j}{ }^{ \pm}\right) \cdot\left|a_{i(v) j^{ \pm}}\right|^{+} \cdot\left(x_{j^{*}}{ }^{-}-x_{j \mathrm{opt}}{ }^{-} \cdot r_{i(v)}\right)\right)$

$\leq b_{i(v)}^{+} \cdot\left(1-r_{i(v)}\right)$ for any $v \in \boldsymbol{V}$

$x_{j^{*}}{ }^{+} \geq x_{j o p t}{ }^{-}$and $x_{j^{*}}{ }^{+} \leq x_{\text {jopt }}{ }^{+}$for any $j \in J_{1}$

$x_{j^{*}}{ }^{-} \geq x_{j o p t}{ }^{-}$and $x_{j^{*}} \leq x_{j o p t}{ }^{+}$for any $j \in J_{2}$.

Model (5) is a linear programming model where all coefficients are deterministic rather than interval sets. The simplex method (Dantzig, 1963) is employed again for solving $L P$ model (5). Let the obtained solutions be denoted as $x_{j o p t *}{ }^{+}\left(j \in J_{1}\right)$ and $x_{\text {jopt* }}{ }^{-}\left(j \in J_{2}\right)$. The objective function (5-1), denoted as $F_{\text {opt* }^{*}}$, equals to $F_{\text {opt }^{*}}{ }^{+}=\sum_{j \in J_{1}}\left(c_{j}^{+} \cdot x_{j \text { opt }^{*}}\right)+\sum_{j \in J_{2}}\left(c_{j}^{+} \cdot x_{\text {jopt* }}{ }^{-}\right)$. Accordingly, the optimistic solution is contracted from $x_{\text {opt }}{ }^{+}(j \in$ $\left.\boldsymbol{J}_{1}\right)$ and $x_{\text {jopt }}{ }^{-}\left(j \in \boldsymbol{J}_{2}\right)$ to $x_{\text {jopt }}{ }^{+}\left(j \in \boldsymbol{J}_{1}\right)$ and $x_{\text {jopt }}{ }^{-}\left(j \in \boldsymbol{J}_{2}\right)$. The maximum objective function value under optimism decreases from $\sum_{j \in J_{1}}\left(c_{j}^{+} \cdot x_{j \mathrm{opt}}{ }^{+}\right)+\sum_{j \in J_{2}}\left(c_{j}^{+} \cdot x_{j \mathrm{opt}}{ }^{-}\right)$to $\sum_{j \in J_{1}}\left(c_{j}^{+} \cdot x_{j \mathrm{opt}}{ }^{+}\right)+\sum_{j \in J_{2}}\left(c_{j}^{+}\right.$. $\left.x_{\text {jopt }} *^{-}\right)$for mitigating violation of constraints (2-2) to (2-4).

\subsection{Contraction of Conservative Solutions}

Based on solutions from the third sub-model, another submodel (denoted as ILP4) for contracting conservative solutions obtained from the second sub-model (ILP2) can be formulated as follows:

$\min F_{*}^{-}=\sum_{j \in J_{1}}\left(c_{j}^{-} \cdot x_{j^{*}}\right)+\sum_{j \in J_{2}}\left(c_{j}^{-} \cdot x_{j^{*}}\right)$

subject to

$$
\begin{aligned}
& S R A_{i(v) 1} \geq r_{i(v)} \text { for any } v \in V \\
& x_{j^{*}} \in x_{j \mathrm{opt}}{ }^{ \pm} \text {for any } j \in J_{1} \\
& x_{j^{*}}{ }^{+} \in x_{j \mathrm{opt}}{ }^{ \pm} \text {for any } j \in J_{2} \\
& x_{j^{*}} \leq x_{j \mathrm{opt} *^{+}} \text {for any } j \in J_{1}
\end{aligned}
$$

$x_{j^{*}}{ }^{+} \geq x_{j o p t *}{ }^{-}$for any $j \in J_{2}$

where $x_{j^{*}}\left(j \in \boldsymbol{J}_{1}\right)$ and $x_{j^{*}}{ }^{+}\left(j \in \boldsymbol{J}_{2}\right)$ are decision variables and $S R A_{i(v) 1}(v \in V)$ are contraction ratios for any violated constraint. Equivalently, sub-model ILP4 can be formulated as:

$\min F_{*}^{-}=\sum_{j \in J_{1}}\left(c_{j}^{-} \cdot x_{j^{*}}\right)+\sum_{j \in J_{2}}\left(c_{j}^{-} \cdot x_{j^{*}}{ }^{+}\right)$

subject to

$\sum_{j \in J_{1}}\left(\operatorname{SIGp}\left(a_{i(v) j}{ }^{ \pm}\right) \cdot\left|a_{i(v) j}\right|^{-} \cdot x_{j \mathrm{opt}}{ }^{+} \cdot r_{i(v)}\right)$

$+\sum_{j \in J_{1}}\left(\operatorname{SIGn}\left(a_{i(v) j^{ \pm}}\right) \cdot\left|a_{i(v) j^{ \pm}}\right|^{-} \cdot\left(x_{\mathrm{jopt}}{ }^{-} \cdot r_{i(v)}-x_{j \mathrm{opt}}{ }^{-}+x_{j^{*}}{ }^{-}\right)\right)$

$+\sum_{j \in J_{2}}\left(\operatorname{SIGp}\left(a_{i(v) j}{ }^{ \pm}\right) \cdot\left|a_{i(v) j}\right|^{+} \cdot\left(x_{j \mathrm{opt}}{ }^{+} \cdot r_{i(v)}-x_{j \mathrm{opt}}{ }^{+}+x_{j^{*}}{ }^{+}\right)\right)$

$+\sum_{j \in J_{2}}\left(\operatorname{SIGn}\left(a_{i(v) j^{ \pm}}\right) \cdot\left|a_{i(v) j}\right|^{+} \cdot x_{j \mathrm{opt}}^{-} \cdot r_{i(v)}\right)$

$\leq b_{i(v)}{ }^{+} \cdot r_{i(v)}$ for any $v \in \boldsymbol{V}$

$x_{\text {jopt }}{ }^{-} \leq x_{j^{*}}{ }^{-} \leq x_{\text {jopt* }}{ }^{+}$for any $j \in J_{1}$

$x_{\text {jopt }}{ }^{+} \geq x_{j^{*}}{ }^{+} \geq x_{\text {jopt* }}{ }^{-}$for any $j \in J_{2}$.

The simplex method (Dantzig, 1963) is employed again to solve $L P$ model (7). Let the obtained solutions be denoted as $x_{\text {jopt* }}{ }^{-}\left(j \in J_{1}\right)$ and $x_{\text {opt }^{*}}{ }^{+}\left(j \in \boldsymbol{J}_{2}\right)$. The objective function $\left(F_{\mathrm{opt}^{*}}\right)$ equals to $\sum_{j \in J_{1}}\left(c_{j}^{-} \cdot x_{j \mathrm{opt} *}{ }^{-}\right)+\sum_{j \in J_{2}}\left(c_{j}^{-} \cdot x_{j \mathrm{opt}} *^{+}\right)$. As a result, the conservative solution is contracted from $x_{j \mathrm{opt}}{ }^{-}\left(j \in J_{1}\right)$ and $x_{\text {jopt }}{ }^{+}(j \in$ $\left.\boldsymbol{J}_{2}\right)$ to $x_{j \mathrm{opt}^{*}}\left(j \in \boldsymbol{J}_{1}\right)$ and $x_{j \mathrm{opt} *^{+}}\left(j \in \boldsymbol{J}_{2}\right)$. As another cost of mitigating constraint violation of ILP models, the maximum objective function value under conservative conditions decreases from $\sum_{j \in J_{1}}\left(c_{j}^{-} \cdot x_{j \mathrm{opt}}{ }^{-}\right)+\sum_{j \in J_{2}}\left(c_{j}^{-} \cdot x_{j \mathrm{opt}}{ }^{+}\right)$to $\sum_{j \in J_{1}}\left(c_{j}^{-} \cdot x_{j \mathrm{opt}}{ }^{-}\right)+\sum_{j \in J_{2}}$ $\left(c_{j}^{-} \cdot x_{j \mathrm{opt} *^{+}}\right)$.

\subsection{Finalization of Decision Space}

Corresponding to a given set of contraction ratios, interval-set solutions are obtained through combining crisp solutions from the third and fourth linear programming sub-models, i.e., $\left\{x_{j \mathrm{opt} *^{*}} \mid x_{\text {jopt* }}{ }^{ \pm}=\left[x_{\text {jopt }}{ }^{-}, x_{j \mathrm{opt} *}{ }^{+}\right] ; j \in \boldsymbol{J}\right\}$. The range of objective function $F_{\text {opt* }^{*}}{ }^{*}$ is a closed interval $\left[F_{\text {opt }^{*}}{ }^{-}, F_{\text {opt }^{*}}{ }^{+}\right.$. Let the final decision space after contraction be denoted as $C D S$. We can have $C D S=\left\{x_{j \mathrm{opt} *} \mid x_{j \mathrm{opt}} \in\left[x_{j \mathrm{opt} *^{*}}, x_{j \mathrm{opt} *^{*}}\right] ; j \in \boldsymbol{J}\right\}$.

The decision space $(C D S)$ is projected as an interval (i.e., $\left.\left[x_{\text {jopt }}{ }^{-}, x_{\text {jopt }}{ }^{+}\right]\right)$on the $j$ th $(j \in J)$ dimension. In engineering practices, this interval can provide decision makers with an initial estimation of desired ranges of the $j$ th decision (i.e., the $j$ th decision variable $x_{j}$ ) in a visualized way. As stated in section 2 , the largest continuous decision space (DILPMax) of ILP models is an irregular multi-dimension space. It is not easily visualized by decision makers especially when a number of decision variables are involved. In comparison with $C D S$, less valuable information can be provided from DILPMax for decision makers even when only a few of decision variables are involved. Another potential substitute of IDS and DILPMax is the hypercube space that can cover DILPMax and of which the projection on any dimension is an interval. Let it be denoted as IDSMax. However, decision space IDSMax may involve many absolutely infeasible solutions that are infeasible for any combination of coefficients. The stability of real-world management systems 


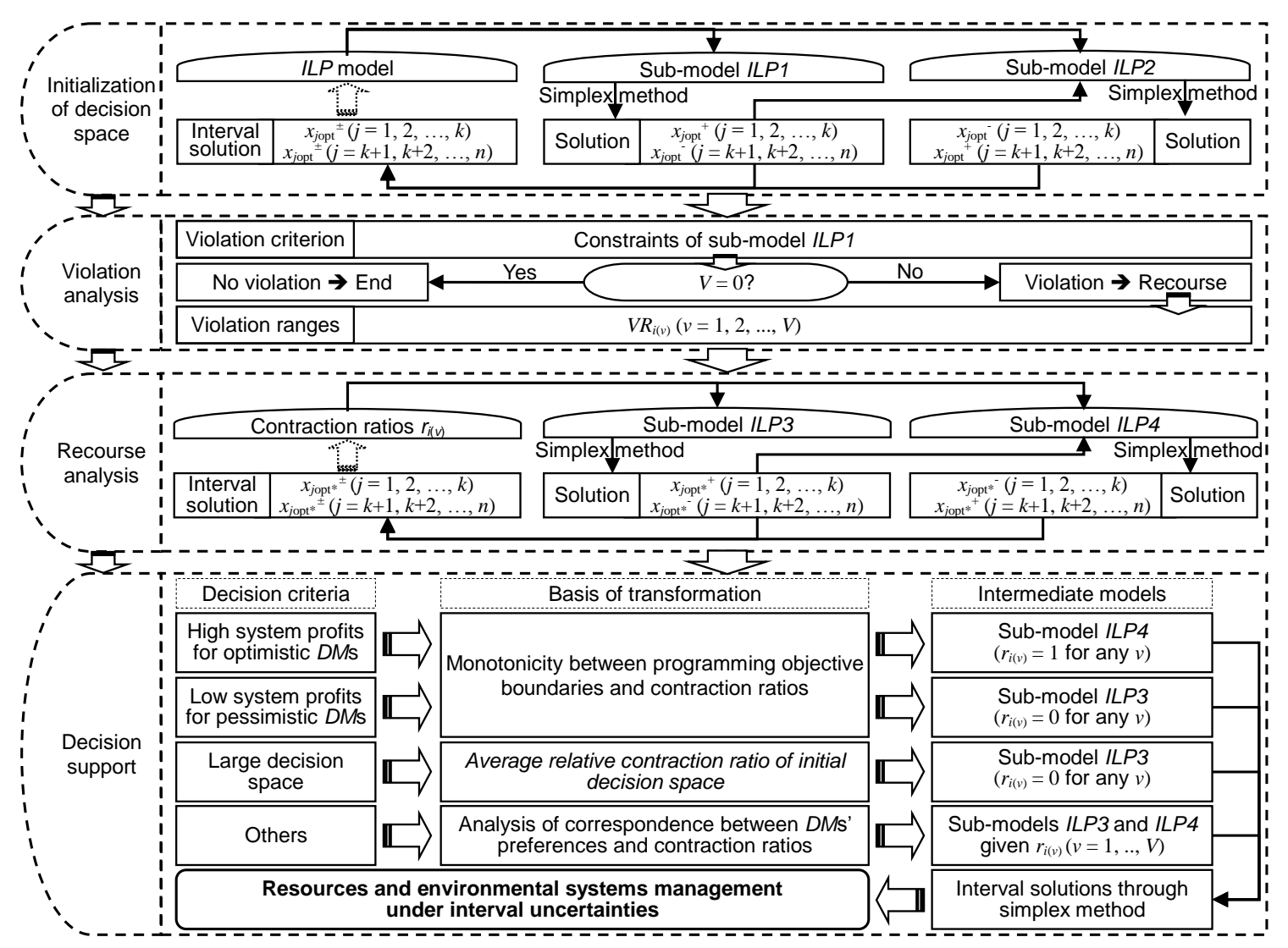

Figure 1. Structure and procedures of the IRLP approach for ILP models.

corresponding to ILP models is decreased if management schemes are determined on the basis of IDSMax.

The decision space after contraction provides a potential to analyze the tradeoff between system optimality and constraint violation of ILP models. The existence of this tradeoff is due to interval uncertainties of inexact coefficients. Decisions approaching $\left(x_{\text {lopt }}{ }^{+}, x_{2 \mathrm{opt}}{ }^{+}, \ldots, x_{\mathrm{kopt}^{+}}{ }^{+}, x_{(k+1) \mathrm{opt}}{ }^{-}, x_{(k+2) \mathrm{opt}}{ }^{-}, \ldots, x_{n \mathrm{opt}}{ }^{-}\right)$ in $C D S$ would lead to high system profits while taking high chances of constraint violation as the fluctuation of interval coefficients. If decisions around $\left(x_{1 \mathrm{opt}}{ }^{-}, x_{2 \mathrm{opt}}{ }^{-}, \ldots, x_{\mathrm{kopt}}{ }^{-}, x_{(k+1) \mathrm{opt}}{ }^{+}\right.$, $\left.x_{(k+2) \text { opt }}{ }^{+}, \ldots, x_{n o p t}{ }^{+}\right)$in $C D S$ are executed the likeliness of constraint violation would decrease at the cost of decreased system profits. A quantitative analysis of this tradeoff may be enabled through Fourier amplitude sensitivity test (Saltelli and Bolado, 1998), factorial analysis (Jaccard, 1998), multi-criteria assessment (Linkov et al., 2006), or other post-optimality techniques in the future studies. The most desired deterministic decision scheme which may be located at a balanced point in $C D S$ will be provided for decision makers for whom acceptance levels of system optimality and constraint violation are varied.

To sum up, the proposed IRLP approach consists of three steps as presented in the Figure 1. An initial interval-set solution of ILP model (1) is obtained through two correlated linear programming sub-models. Violation analysis is conducted to identify violated constraints. Given a set of constraint contraction ratios, two linear programming sub-models are formulated. The simplex method is employed twice for obtaining interval-set solutions as a contraction of initialized solutions.

\section{Post-Optimality Analysis}

\subsection{Analysis of Interactions}

As a resource measure to eradicate constraint violation problems of initial solutions from sub-models ILPI and ILP2, sub-models ILP3 and ILP4 are proposed in the IRLP approach. The two sub-models can avoid high penalties resulting from violation of constraints in sub-model ILPI while remaining optimality of decision alternatives after resource. This capability relies on designs of sub-models ILP3 and ILP4. Constraints of both sub-models reflect the target of mitigating constraint violation through contracting the initial decision space, and objecttive functions aim to maximize the final decision space after contraction and avoid neglecting potential decision alternatives and resultant system optimality. It should be noted that formulations of the two sub-models can be in other forms. For instance, sub-models ILP3 and ILP4 can be constructed as:

$\min F_{*}^{-}=\sum_{j \in J_{1}}\left(c_{j}^{-} \cdot x_{j^{*}}\right)+\sum_{j \in J_{2}}\left(c_{j}^{-} \cdot x_{j^{*}}{ }^{+}\right)$

subject to

$\sum_{j \in J_{1}}\left(\operatorname{SIGp}\left(a_{i(v) j}{ }^{ \pm}\right) \cdot\left|a_{i(v) j}\right|^{-} \cdot x_{j \mathrm{opt}}{ }^{+} \cdot r_{i(v)}\right)$ 
$+\sum_{j \in J_{1}}\left(\operatorname{SIGn}\left(a_{i(v) j}{ }^{ \pm}\right) \cdot\left|a_{i(v) j}\right|^{ \pm} \cdot \cdot\left(x_{j \mathrm{opt}}{ }^{-} \cdot r_{i(v)}-x_{j \mathrm{opt}}{ }^{-}+x_{j^{*}}{ }^{-}\right)\right)$

$+\sum_{j \in J_{2}}\left(\operatorname{SIGp}\left(a_{i(v) j}{ }^{ \pm}\right) \cdot\left|a_{i(v) j}{ }^{ \pm}\right|^{+} \cdot\left(x_{j \mathrm{opt}}{ }^{+} \cdot r_{i(v)}-x_{j \mathrm{opt}}{ }^{+}+x_{j^{*}}{ }^{+}\right)\right)$

$+\sum_{j \in J_{2}}\left(\left.\operatorname{SIGn}\left(a_{i(v) j}{ }^{ \pm}\right) \cdot\left|a_{i(v) j}\right|^{+}\right|^{+} \cdot x_{j \mathrm{opt}}{ }^{-} \cdot r_{i(v)}\right)$

$\leq b_{i(v)}{ }^{+} \cdot r_{i(v)}$ for any $v \in \boldsymbol{V}$

$x_{\text {jopt }}{ }^{-} \leq x_{j^{*}}{ }^{-} \leq x_{\text {jopt }}{ }^{+}$for any $j \in J_{1}$

$x_{\text {jopt }}{ }^{+} \geq x_{j^{*}}{ }^{+} \geq x_{\text {jopt }}{ }^{-}$for any $j \in J_{2}$

and

$\max F_{*}^{+}=\sum_{j \in J_{1}}\left(c_{j}^{+} \cdot x_{j^{*}}^{+}\right)+\sum_{j \in J_{2}}\left(c_{j}^{+} \cdot x_{j^{*}}\right)$

subject to

$\sum_{j \in J_{1}}\left(\operatorname{SIGp}\left(a_{i(v) j^{ \pm}}\right) \cdot\left|a_{i(v) j j^{ \pm}}\right|^{-} \cdot\left(x_{j^{*}}{ }^{+}-x_{j \mathrm{opt}}{ }^{+} \cdot r_{i(v)}\right)\right)$

$+\sum_{j \in J_{1}}\left(\operatorname{SIGn}\left(a_{i(v) j j^{ \pm}}\right) \cdot\left|a_{i(v) j}\right|^{-} \cdot x_{j \mathrm{opt}}{ }^{-} \cdot\left(1-r_{i(v)}\right)\right)$

$+\sum_{j \in J_{2}}\left(\operatorname{SIGp}\left(a_{i(v) j}{ }^{ \pm}\right) \cdot\left|a_{i(v) j}\right|^{+} \cdot x_{j \mathrm{opt}}{ }^{+} \cdot\left(1-r_{i(v)}\right)\right)$

$+\sum_{j \in J_{2}}\left(\operatorname{SIGn}\left(a_{i(v) j^{ \pm}}\right) \cdot\left|a_{i(v) j j^{ \pm}}\right|^{+} \cdot\left(x_{j^{*}}{ }^{-}-x_{j \mathrm{opt}}{ }^{-} \cdot r_{i(v)}\right)\right)$

$\leq b_{i(v)}{ }^{+} \cdot\left(1-r_{i(v)}\right)$ for any $v \in \boldsymbol{V}$

$x_{\text {jopt }{ }^{*}} \leq x_{j^{*}}{ }^{+} \leq x_{j \mathrm{opt}}{ }^{+}$for any $j \in \boldsymbol{J}_{1}$

$x_{j \mathrm{opt}}{ }^{-} \leq x_{j^{*}}{ }^{-} \leq x_{j \mathrm{opt}^{*}}{ }^{+}$for any $j \in \boldsymbol{J}_{2}$

respectively, where $x_{1 \mathrm{opt}^{*}}, x_{2 \mathrm{opt} *^{*}}, \ldots, x_{\mathrm{kopt}^{*}}{ }^{-}, x_{(k+1) \mathrm{opt} *^{*}}, x_{(k+2) \mathrm{opt} *^{*}}$, ..., and $x_{n \mathrm{opt}^{*}}{ }^{+}$are optimal solutions of $L P$ model (8) through the simplex method (Dantzig, 1963). The above formulations of sub-models ILP3 and ILP4 can also effectively resolve constraint violation problems of the TSA approach through contraction of the initial decision space. It is possible that solutions of models (8) and (9) are identical with those of models (5) and (7). On the other hand, it may hold in some cases that optimal values of objective functions $F_{*}{ }^{-}$and $F_{*}{ }^{+}$in models (8) and (9) are decreased in comparison with $F_{\text {opt }^{*}}{ }^{-}$and $F_{\text {opt*}^{*}}{ }^{+}$in models (7) and (5). Potentially high system profits may be neglected although the constraint violation risk is decreased simultaneously. Results of models (8) and (9) are relatively conservative. Formulating sub-models ILP3 and ILP4 as models (8) and (9) is preferable for decision makers who do not pursue the highest system profit. Based on the assumption that provision of the highest system profit is desired for most decision makers, submodels ILP3 and ILP4 are formulated as models (5) and (7) in this study, respectively.

Proposition 2: If $S R_{i(v) 1}=V R_{i(v)}$ for any $v \in V$, then $F_{\text {opt }^{*}}$ $=F_{\text {opt }}{ }^{+}$; if $V R_{i(v)} \leq M S R_{2 i(v)}$ and $S R_{i(v) 2}=V R_{i(v)}$ for any $v \in V$, then $F_{\text {opt* }^{-}}=F_{\text {opt }^{-}}$.

Proof: Since the formulation of $S R A_{i(v) 1}$, we have $S R A_{i(v) 1}=$ 1 if $S R_{i(v) 1}=V R_{i(v)}$ for any $v \in V$. From $S R A_{i(v) 1}+S R A_{i(v) 2} \geq 1$, we have $S R A_{i(v) 2} \geq 0$. Sub-model ILP3 is simplified as Max $F_{*}{ }^{+}$ $=\sum_{j \in J_{1}}\left(c_{j}^{+} \cdot x_{j *^{+}}\right)+\sum_{j \in J_{2}}\left(c_{j}^{+} \cdot x_{j^{*}}\right)$ subject to $S R A_{i(v) 2} \geq 0$ for any $v$ $\in \boldsymbol{V}, x_{j^{*}}{ }^{+} \in x_{\text {jopt }}{ }^{ \pm}$for any $j \in \boldsymbol{J}_{1}$, and $x_{j^{*}}{ }^{-} \in x_{\text {jopt }}{ }^{{ }^{*}}$ for any $j \in \boldsymbol{J}_{2}$. It is equivalent with maximizing $\left\{F_{*^{+}}=\sum_{j \in J_{1}}\left(c_{j}^{+} \cdot x_{j^{*}}\right)+\sum_{j \in J_{2}}\left(c_{j}^{+}\right.\right.$. $\left.x_{j^{*}}{ }^{-}\right) \mid x_{j *^{+}} \in x_{\text {jopt }}{ }^{ \pm}$for any $j \in \boldsymbol{J}_{1} ; x_{j^{*}} \in x_{\text {jopt }}{ }^{ \pm}$for any $\left.j \in \boldsymbol{J}_{2}\right\}$ due to non-negativity of $S R A_{i(v) 2}$ according to its definition. The optimal solution of the simplified crisp model is $\left\{x_{1 \mathrm{opt}^{+}}{ }^{+}, x_{2 \mathrm{opt}}{ }^{+}, \ldots\right.$,

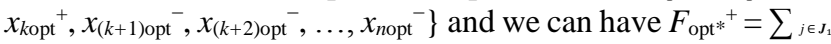

$\left(c_{j}^{+} \cdot x_{j o p t}{ }^{+}\right)+\sum_{j \in J_{2}}\left(c_{j}^{+} \cdot x_{j o p t}{ }^{-}\right)$. From the formulation of $F_{\text {opt }^{+}}$, we have $F_{\text {opt }^{*}}{ }^{+}=F_{\text {opt }}{ }^{+}$. Similarly, sub-model ILP4 is equivalent with minimizing $\left\{F_{*}^{-}=\sum_{j \in J_{1}}\left(c_{j}^{-} \cdot x_{j^{*}}\right)+\sum_{j \in J_{2}}\left(c_{j}^{-} \cdot x_{j^{*}}\right) \mid S R A_{i(v) 1} \geq 0\right.$ for any $v \in V ; x_{j o p t}{ }^{-} \leq x_{j^{*}}{ }^{-} \leq x_{j \mathrm{opt}{ }^{*}}{ }^{+}$for any $j \in J_{1} ; x_{j \mathrm{opt}}{ }^{+} \geq x_{j^{*}}{ }^{+} \geq$ $x_{\text {jopt* }}{ }^{-}$for any $\left.j \in J_{2}\right\}$ if $V R_{i(v)} \leq M S R_{2 i(v)}$ and $S R_{i(v) 2}=V R_{i(v)}$. Thus, $F_{\mathrm{opt}^{*}}=\sum_{j \in J_{1}}\left(c_{j}^{-} \cdot x_{\text {jopt }}{ }^{-}\right)+\sum_{j \in J_{2}}\left(c_{j}^{-} \cdot x_{j \mathrm{opt}}{ }^{+}\right)$and $F_{\mathrm{opt}^{*}}{ }^{-}=F_{\mathrm{opt}}{ }^{-}$.

Proposition 3: Let $r_{i(v) 1}$ and $r_{i(v) 2}$ be two contraction ratios given for the $v$ th violated constraint. If $r_{i(v) 1} \leq r_{i(v) 2}$, then $F_{\text {opt } 1^{*}}$ $\leq F_{\text {opt } 2 *}{ }^{-}$and $F_{\text {opt } 1 *}{ }^{+} \leq F_{\text {opt } 2 *}{ }^{+}$where $F_{\text {opts }^{*}}$ and $F_{\text {opts }} *^{+}(s=1$ or 2$)$ are the lower and upper bounds of optimal objective function values under contraction ratio $r_{i(v) s}$, respectively.

Proof: Denote the feasible region of sub-model ILP3 as $F R 3_{1}$ and $F R 3_{2}$ corresponding to $r_{i(v) 1}$ and $r_{i(v) 2}$, respectively. We can have $F R 3_{1}=\left\{\left(x_{1}, x_{2}, \ldots, x_{k}, x_{(k+1)}, x_{(k+2)}, \ldots, x_{n}\right) \mid S R A_{i(v) 2} \geq\right.$ $1-r_{i(v) 1}$ for any $v \in V ; x_{j} \in x_{j \text { opt }}{ }^{ \pm}$for any $j \in J_{1} ; x_{j} \in x_{j o p t}{ }^{ \pm}$for any $\left.j \in \boldsymbol{J}_{2}\right\}$ and $F R 3_{2}=\left\{\left(x_{1}, x_{2}, \ldots, x_{k}, x_{(k+1)}, x_{(k+2)}, \ldots, x_{n}\right) \mid S R A_{i(v) 2}\right.$ $\geq 1-r_{i(v) 2}$ for any $v \in V ; x_{j} \in x_{\text {jopt }}{ }^{ \pm}$for any $j \in J_{1} ; x_{j} \in x_{\text {jopt }}{ }^{ \pm}$for any $\left.j \in \boldsymbol{J}_{2}\right\}$. Suppose $\left\{x_{11^{*}}{ }^{+}, x_{211^{+}}, \ldots, x_{k 11^{*}}, x_{(k+1) 1^{*}}, x_{(k+2) 1 *^{-}}, \ldots\right.$, $\left.x_{n 1} *^{-}\right\}$is any feasible solution of sub-model ILP3 under contraction ratios $r_{i(v) 1}$. Let it be abbreviated as $\boldsymbol{X}_{1 *}$. Equivalently, $\boldsymbol{X}_{1 *}$ $\in F R 3_{1}$. As for constraint (5-2), we have $\sum_{j \in J_{1}}\left(\operatorname{SIGp}\left(a_{i(v) j}\right)^{ \pm}\right.$. $\left.\left|a_{i(v) j}\right|^{ \pm} \cdot\left(x_{j o p t}{ }^{+}-x_{j 1 *^{+}}\right)\right)+\sum_{j \in J_{2}}\left(\left.\operatorname{SIGn}\left(a_{i(v) j}{ }^{ \pm}\right) \cdot\left|a_{i(v) j}\right|^{ \pm}\right|^{+} \cdot\left(x_{j o p t}{ }^{-}-x_{j 11^{*}}\right)\right)$ $\geq V R_{i(v)}-V R_{i(v)} \cdot r_{i(v) 1}$. Since $0 \leq r_{i(v) 1} \leq r_{i(v) 2} \leq 1$ and $V R_{i(v)} \geq 0$, we have $V R_{i(v)}-V R_{i(v)} \cdot r_{i(v) 1} \geq V R_{i(v)}-V R_{i(v)} \cdot r_{i(v) 2}$. Accordingly, $\sum_{j \in J_{1}}$ $\left.\left(\operatorname{SIGp}\left(a_{i(v) j}\right)^{ \pm}\right) \cdot\left|a_{i(v) j}\right|^{-} \cdot\left(x_{j \mathrm{opt}}{ }^{+}-x_{j 1^{*}}{ }^{+}\right)\right)+\sum_{j \in J_{2}}\left(\operatorname{SIGn}\left(a_{i(v) j^{ \pm}}\right) \cdot\left|a_{i(v) j}\right|^{+}\right.$ $\left.\cdot\left(x_{j \text { opt }}{ }^{-}-x_{j 11^{*}}\right)\right) \geq V R_{i(v)}-V R_{i(v)} \cdot r_{i(v) 2}$. We can have $X_{1 *} \in F R 3_{2}$ since the only difference of constraints between feasible regions $F R 3_{1}$ and $F R 3_{2}$ is constraint (5-2). Thus, $F R 3_{1} \subseteq F R 3_{2}$. Accordingly, we can have $F_{\mathrm{opt}^{*}{ }^{+}} \leq F_{\mathrm{opt}^{*}}{ }^{+}$because of the enlarged feasible region under contraction ratio $r_{i(v) 2}$. Similarly, we can have the feasible region of sub-model ILP4 is shrunk as the contraction ratio increases from $r_{i(v) 1}$ to $r_{i(v) 2}$. Thus, the optimality of objective function (6-1) or (7-1) is reduced. Accordingly, we have $F_{\mathrm{opt1}^{*}} \leq F_{\mathrm{opt}^{*}{ }^{-}}$.

\subsection{Tradeoff Analysis}

In the proposed $I R L P$ approach, potential violation of constraints of sub-model ILPI is mitigated through contracting optimistic decisions from sub-model ILPI or conservative decision from sub-model ILP2. Contraction of either group of decisions is helpful for coping with constraint-violation problems. There is a tradeoff between contraction ranges of two groups of decisions. The tradeoff is reflected as values of contraction ratios. Under higher contraction ratios, mitigation of constraint violation is contributed more by contraction of conservative decisions. Namely, more conservative decisions in the initial decision space are eradicated, and optimistic decisions are remained. This strategy is preferable for optimistic decision makers other than conservative ones. In contrast, as contraction ratios decrease from one to zero, contraction ranges of optimistic decisions climb while those of conservative decisions drop. Mitigation of constraint violation is achieved more by contraction of optimistic decisions. Retention of more conservative decisions can help conservative decision makers identify a more desired management scheme although this is beyond expectations of optimistic decision makers. The IRLP approach can be simp- 
lified under particular scenarios due to diversity of preferences of decision makers.

For the most optimistic decision makers, contraction ratio $r_{i(v)}$ approaches 1 for all violated constraints. In this case, there are only three sub-models, i.e., ILP1, ILP2 and ILP4. In this case, sub-model ILP4 can be simplified as:

$\min F_{*}^{-}=\sum_{j \in J_{1}}\left(c_{j}^{-} \cdot x_{j^{*}}{ }^{-}\right)+\sum_{j \in J_{2}}\left(c_{j}^{-} \cdot x_{j^{*}}{ }^{+}\right)$

subject to

$$
\begin{aligned}
& \sum_{j \in J_{1}}\left(\operatorname{SIGp}\left(a_{i(v) j} j^{ \pm}\right) \cdot\left|a_{i(v) j}\right|^{ \pm} \cdot x_{j \mathrm{opt}}{ }^{+}\right) \\
& +\sum_{j \in J_{1}}\left(\left.\operatorname{SIGn}\left(a_{i(v) j} j^{ \pm}\right) \cdot\left|a_{i(v) j}\right|^{ \pm}\right|^{-} \cdot\left(x_{j \mathrm{opt}}{ }^{-}-x_{j \mathrm{opt}}{ }^{-}+x_{j^{*}}{ }^{-}\right)\right) \\
& +\sum_{j \in J_{2}}\left(\operatorname{SIGp}\left(a_{i(v) j j^{ \pm}}\right) \cdot\left|a_{i(v) j}{ }^{+}\right|^{+} \cdot\left(x_{j \mathrm{opt}}{ }^{+}-x_{j \mathrm{opt}}{ }^{+}+x_{j^{*}}{ }^{+}\right)\right) \\
& +\sum_{j \in J_{2}}\left(\operatorname{SIGn}\left(a_{i(v) j^{ \pm}}\right) \cdot\left|a_{i(v) j^{ \pm}}\right|^{+} \cdot x_{j \mathrm{opt}}{ }^{-}\right) \\
& \leq b_{i(v)}{ }^{+} \text {for any } v \in \boldsymbol{V} \\
& x_{\text {jopt }}{ }^{-} \leq x_{j^{*}}{ }^{-} \leq x_{\text {jopt }}{ }^{+} \text {for any } j \in J_{1} \\
& x_{\text {jopt }}{ }^{+} \geq x_{j^{*}}{ }^{+} \geq x_{\text {jopt }}{ }^{-} \text {for any } j \in J_{2}
\end{aligned}
$$

where $x_{j^{*}}\left(j \in \boldsymbol{J}_{1}\right)$ and $x_{j^{*}}{ }^{+}\left(j \in \boldsymbol{J}_{2}\right)$ are decision variables and $x_{\text {jopt }}{ }^{ \pm}(j \in J)$ are initialized interval-set solutions obtained from sub-models $I L P I$ and $I L P 2$. However, the initial decision space composed of $x_{\text {jopt }}{ }^{ \pm}(j \in J)$ may be wasted in some cases. Assume the first $k$ interval-set coefficients in the $i(v)$ th $(v \in V)$ constraint, i.e., $a_{i(v) 1} 1^{ \pm}, a_{i(v) 2^{ \pm}}, \ldots$ and $a_{i(v) k}{ }^{ \pm}$, are negative; the last $n-k$ interval-set coefficients, i.e., $a_{i(v)(k+1)^{ \pm}}, a_{i(v)(k+2)^{ \pm}}, \ldots$ and $a_{i(v) n}{ }^{ \pm}$, are nonnegative; and $\left(\boldsymbol{A}_{i(v)}{ }^{ \pm} \boldsymbol{X}^{ \pm}\right)_{\mathrm{C}} \leq b_{i(v)}{ }^{+}$. We have $\operatorname{SIGp}\left(a_{i(v) j}{ }^{ \pm}\right)=$ 0 and $\left.\operatorname{SIGn}\left(a_{i(v) j}\right)^{ \pm}\right)=-1$ hold for any $j \in J_{1}$, and $\operatorname{SIGp}\left(a_{i(v) j} \pm\right)=1$ and $\operatorname{SIGn}\left(a_{i(v) j}{ }^{ \pm}\right)=0$ hold for any $j \in \boldsymbol{J}_{2}$. The final interval-set solution $\left(I F S_{1(v)^{ \pm}}\right)$after contraction through the first method is degenerated into a vector of real numbers, i.e., $\left(x_{1 \mathrm{opt}}{ }^{+}, x_{2 \mathrm{opt}}{ }^{+}, \ldots\right.$, $\left.x_{\text {kopt }}{ }^{+}, x_{(k+1) \mathrm{opt}^{-}}, x_{(k+2) \mathrm{opt}^{-}}, \ldots, x_{n \mathrm{opt}}\right)^{\mathrm{T}}$. The degenerated solution corresponds to the upper bound of optimal objective-function values, i.e., $F^{+}=\sum_{j \in J_{1}}\left(c_{j}^{+} \cdot x_{j}^{+}\right)+\sum_{j \in J_{2}}\left(c_{j}^{+} \cdot x_{j}^{-}\right)$. It can only be provided for optimistic decision makers other than conservative ones, since undertaking high constraint-violation risks is necessary.

On the other hand, the most conservative decision makers may expect that contraction ratio $r_{i(v)}$ equals to 0 for any violated constraint $v \in V$. In this case, sub-model ILP4 would be eliminated. There would be just sub-models $I L P 1, I L P 2$ and ILP3. Sub-model ILP3 is equivalent to the following linear programming model:

$\max F_{*}^{+}=\sum_{j \in J_{1}}\left(c_{j}^{+} \cdot x_{j^{*}}^{+}\right)+\sum_{j \in J_{2}}\left(c_{j}^{+} \cdot x_{j^{*}}\right)$

subject to

$$
\begin{aligned}
& \sum_{j \in J_{1}}\left(\operatorname{SIGp}\left(a_{i(v) j}{ }^{ \pm}\right) \cdot\left|a_{i(v) j j^{ \pm}}\right|^{-} \cdot x_{j^{*}}{ }^{+}\right) \\
& +\sum_{j \in J_{1}}\left(\operatorname{SIGn}\left(a_{i(v) j}{ }^{ \pm}\right) \cdot\left|a_{i(v) j}\right|^{ \pm} \cdot x_{j \mathrm{opt}}{ }^{-}\right) \\
& +\sum_{j \in J_{2}}\left(\operatorname{SIGp}\left(a_{i(v) j}{ }^{ \pm}\right) \cdot\left|a_{i(v) j}\right|^{+} \cdot x_{j \mathrm{opt}}{ }^{+}\right) \\
& +\sum_{j \in J_{2}}\left(\operatorname{SIGn}\left(a_{i(v) j}{ }^{ \pm}\right) \cdot\left|a_{i(v) j}\right|^{+} \cdot x_{j^{*}}\right) \\
& \leq b_{i(v)}{ }^{+} \text {for any } v \in \boldsymbol{V} \\
& x_{j *^{*}} \geq x_{j o p t}{ }^{-} \text {and } x_{j *^{*}}{ }^{+} \leq x_{j o p t}{ }^{+} \text {for any } j \in J_{1} \\
& x_{j^{*}}{ }^{-} \geq x_{j o p t}{ }^{-} \text {and } x_{j^{*}}{ }^{-} \leq x_{j o p t}{ }^{+} \text {for any } j \in J_{2}
\end{aligned}
$$

where $x_{j^{*}}{ }^{+}\left(j \in \boldsymbol{J}_{1}\right)$ and $x_{j^{*}}\left(j \in \boldsymbol{J}_{2}\right)$ are decision variables and $x_{j o p t}{ }^{ \pm}(j \in J)$ are initialized interval-set solutions obtained from sub-models $I L P I$ and $I L P 2$. The potential challenge is that most of the initial decision space may be cut. In particular cases, it would be degenerated into a point in an $n$-dimensional Euclidean space, i.e., $\left(x_{1 \mathrm{opt}}{ }^{-}, x_{2 \mathrm{opt}}{ }^{-}, \ldots, x_{k \mathrm{opt}}{ }^{-}, x_{(k+1) \mathrm{opt}}{ }^{+}, x_{(k+2) \mathrm{opt}}{ }^{+}, \ldots\right.$, $\left.x_{n \text { opt }}{ }^{+}\right)^{\mathrm{T}}$. The degenerated solution which corresponds to the lower bound of optimal objective-function values $F^{-}=\sum_{j \in J_{1}}\left(c_{j}^{-}\right.$. $\left.x_{j}^{-}\right)+\sum_{j \in J_{2}}\left(c_{j}^{-} \cdot x_{j}^{+}\right)$, is preferred for conservative decision makers but not for optimistic ones. The expectation of high system profits can hardly be satisfied. The interval-set solution (i.e., $\left.I F S_{2 i(v)^{ \pm}}\right)$is conditionally feasible. It is feasible for ILP model (1) and can mitigate the violation problem of constraints (2-2) if and only if $M S R_{2 i(v)} \geq V R_{i(v)}$.

\subsection{Dimensional Analysis of Decision Space}

It is also of possibility that decision makers prefer to a large decision space that includes as many alternatives as possible for management under interval uncertainties. This relies on proposition of an index for quantifying the size of the decision space. Multiple conventional geometric indexes are available, e.g., $\prod X=\prod_{j=1}^{n}\left(x_{j o p *^{*}}-x_{j o p t *}\right)$ or $\sum X=\sum_{j=1}^{n}\left(x_{j o p *^{*}}{ }^{+}-x_{j o p t *} *^{-}\right)$, but they are ineffective for the proposed $I R L P$ approach. The $j$ th interval-set solution, i.e., $x_{\text {jopt }}^{ \pm}(j \in J)$, may be contracted to a real number instead of an interval set, i.e., $x_{\text {jopt }}{ }^{+}=x_{j o p t}{ }^{-}$. Accordingly, index $\prod X$ equals to zero no matter how much value ranges of other decision variables are after contraction. As for in $\operatorname{dex} \sum X$, it can only quantify the absolute size of the final decision space. The connection between initial interval-set solutions, $x_{\text {jopt }}{ }^{ \pm}(j \in \boldsymbol{J})$, and final ones, $x_{\text {jopt } *^{ \pm}}(j \in \boldsymbol{J})$ may be removed if it is employed. In consideration of the process that final solutions $x_{j \text { opt } *^{ \pm}}(j \in J)$ are obtained through contraction of the initial interval-set solutions $\left(x_{j o p t}{ }^{ \pm}, j \in J\right)$, the index of the average relative contraction ratio of the initial decision space is proposed for measuring the size of finalized decision space in this study. It is abbreviated as $D S R$. A higher value of $D S R$ represents a broader decision space, and vice versa.

Definition 3: $D S R=\sum_{s=1}{ }^{S}\left(\left(x_{j(s) \mathrm{opt}^{*}}{ }^{+}-x_{j(s) \mathrm{opt}^{*}}{ }^{-}\right) /\left(x_{j(s) \mathrm{opt}}{ }^{+}-\right.\right.$ $\left.\left.x_{j(s) \text { opt }}{ }^{-}\right)\right) / S$ where $j(s)$ represents type-I decision variables that are interval sets rather than real numbers.

It has been proved above that, when $S R_{i(v) 1}=V R_{i(v)}$ for any $v \in \boldsymbol{V}$, we can have $F_{\text {opt }^{*}}{ }^{+}=F_{\text {opt }}{ }^{+}$. In this case, the contraction ratio equals to one for any $v$. Violation of constraints of submodel ILPI is caused by interval-set solutions of type-I decision variables. Mitigation of this problem is achieved through contracting boundaries of these decision variables while remaining those of type-II decision variables. As a result, $D S R=0$ when $r_{i(v)}=1$ for any $v \in \boldsymbol{V}$, which is verified through an example in the next section. This implies that higher contraction ratios are preferable for decision makers who expect few alternative management schemes. In contrast, others pursuing a large decision space desire as low contraction ratios as possible. Setting $r_{i(v)}$ as zero may be the most advisable option for them. In addition, due to complexities of real-world ILP problems, there may be many other situations such as varied preferences of contraction ranges for violated constraints. It is impossible to cover 
all of them excluding representative ones as discussed above. Adaptation of the proposed IRLP approach to ILP problems under diverse complexities will be examined in the future studies.

\section{Example of REM through IRLP}

An interval-parameter linear $R E M$ problem will be provided to demonstrate advantages of the proposed IRLP method as well as the detailed procedures for the relevant algorithm. Such a problem can be formulated as an ILP model with an objecttive of economic revenue, and constraints of environmental loading capacity and resources recovery:

$\max F^{ \pm}=[50,60] \cdot x_{1}^{ \pm}-[70,90] \cdot x_{2}{ }^{ \pm}$

subject to

$[4,6] \cdot x_{1}^{ \pm}+[1,2] \cdot x_{2}^{ \pm} \leq[140,150]$

$[7,10] \cdot x_{2}^{ \pm}-[1,2] \cdot x_{1}^{ \pm} \geq[1,2]$

$x_{j}^{ \pm} \geq 0$ for $j=1$ and 2

where $x_{1}{ }^{ \pm}$and $x_{2}{ }^{ \pm}$are two interval-set decision variables of which the lower and upper bounds are to be determined; $F^{ \pm}$is a linear function of interval-set coefficients and decision variables. Assumptions 1 to 6 of the IRLP approach are satisfied, e.g., zero is not included in all interval-set coefficients and all decision variables are non-negative.

\subsection{Initialization of Decision Space}

Firstly, ILP model (12) is converted to two correlated linear programming sub-models. Since the objective (12-1) is to maximize value ranges of objective function $F^{ \pm}$, the objective function of the first sub-model should be maximizing the upper bound of objective function $F^{ \pm}$. Since $[50,60] \geq 0,[-90,-70]$ $<0$ and $x_{j}^{ \pm} \geq 0$ for any $j=1$ and 2 , we can have the upper bound of $F^{ \pm}$equals to $60 \cdot x_{1}{ }^{+}-70 \cdot x_{2}^{-}$. Maximization of $60 \cdot x_{1}{ }^{+}-70 \cdot x_{2}$ is equivalent with maximizing $x_{1}{ }^{+}$as well as minimizing $x_{2}^{-}$. Thus, decision variables in the first sub-model are the upper bound of $x_{1}{ }^{ \pm}$and the lower bound of $x_{2}{ }^{ \pm}$. According to formulations of sub-model $I L P I$, the first sub-model corresponding to model (12) can be formulated as:

$\max F^{+}=60 \cdot x_{1}^{+}-70 \cdot x_{2}^{-}$

subject to

$4 \cdot x_{1}{ }^{+}+2 \cdot x_{2}^{-} \leq 150$

$10 \cdot x_{2}^{-}-x_{1}{ }^{+} \geq 1$

$x_{1}^{+} \geq 0$ and $x_{2}^{-} \geq 0$.

As presented in Figure 2, the feasible region constrained by inequalities (13-2) to (13-4) is the parallel-lines-filled area that is under the line $l_{1}$ (i.e., $4 \cdot x_{1}{ }^{+}+2 \cdot x_{2}^{-}=150$ ), above the lines $l_{2}$ (i.e., $10 \cdot x_{2}{ }^{-}-x_{1}{ }^{+}=1$ ) and $x_{2}{ }^{-}=0$, at the right of axis $x_{1}{ }^{+}=0$ and above the axis $x_{2}{ }^{-}=0$. Any combination of $x_{1}{ }^{+}$and $x_{2}{ }^{-}$in this area is feasible for all constraints in model (13). To avoid abnormally high penalties caused by constraint violations, the optimum solution should be located within this area. As shown in Figure 2, the objective function (i.e., line $l_{3}$ ) is maximized at point $p_{1}$. Namely, the optimum solution of model (13) is point $p_{1}$ where $x_{1 \mathrm{opt}}{ }^{+}=35.67$ and $x_{2 \mathrm{opt}}{ }^{-}=3.67$. The upper bound of objective function values (denoated as $F_{\text {opt }^{+}}$) equals to $60 \cdot x_{\text {opt }}{ }^{+}$ $-70 \cdot x_{2 \text { opt }}{ }^{-}$or 1883.33 , which means the optimum value of objective function (13-1) can reach 1883.33 in the best case.

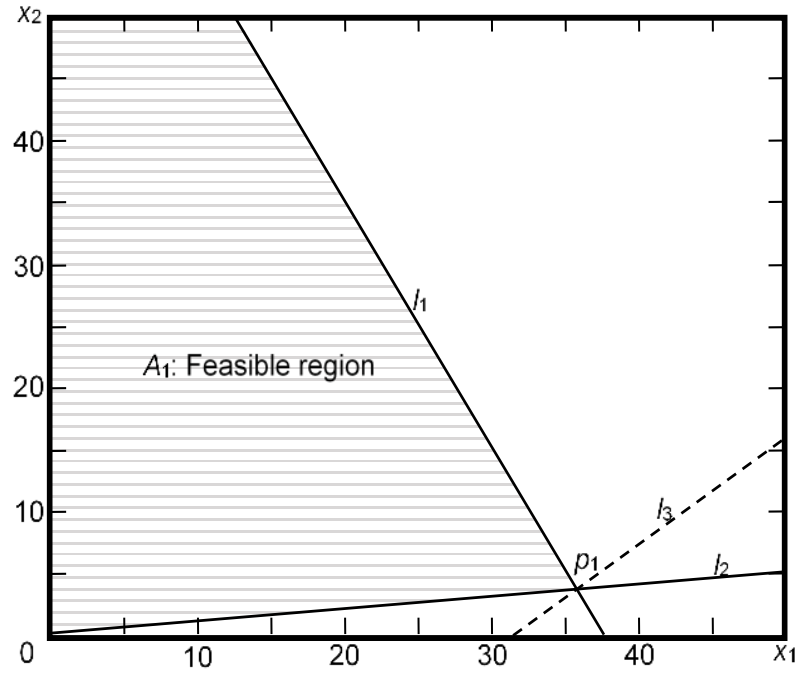

Figure 2. Feasible region and optimum solution of the submodel (13).

The second sub-model is formulated based on solutions of model (13). In contrast to the first sub-model, the objective function of the second one is to maximize the lower bound of objective function (12-1), i.e., Max $F^{-}=50 \cdot x_{1}{ }^{-}-90 \cdot x_{2}{ }^{+}$. To achieve this, the lower bound of $x_{1}{ }^{ \pm}$should be minimized, while the upper bound of $x_{2}{ }^{ \pm}$should be maximized. Thus, decision variables of the second sub-model are the lower bound of $x_{1}{ }^{ \pm}$and the upper bound of $x_{2}{ }^{ \pm}$, which exactly counters to that in the first submodel (13). In accordance with the formulation of model ILP2, the second sub-model is formulated as follows:

$\max F^{-}=50 \cdot x_{1}^{-}-90 \cdot x_{2}{ }^{+}$

subject to

$6 \cdot x_{1}^{-}+x_{2}^{+} \leq 140$

$7 \cdot x_{2}^{+}-2 \cdot x_{1}^{-} \geq 2$

$x_{1}^{-} \geq 0$ and $x_{2}^{+} \geq 0$

$x_{1 \mathrm{opt}}{ }^{+} \geq x_{1}^{-}$and $x_{2}{ }^{+} \geq x_{2 \mathrm{opt}}{ }^{-}$.

The simplex method is employed again for solving deterministic linear programming model (14). The optimal solutions 
are $x_{1 \mathrm{opt}}{ }^{-}=22.23$ and $x_{2 \mathrm{opt}}{ }^{+}=6.64$. As shown in Figure 3, the feasible region constrained by inequalities (14-2) to (14-5) is the parallel-lines-filled area $A_{2}$ that is below the line $l_{4}$ (i.e., 6 . $x_{1}^{-}+x_{2}{ }^{+}=140$ ), above the line $l_{5}$ (i.e., $7 \cdot x_{2}{ }^{+}-2 \cdot x_{1}^{-}=2$ ), at the right of axis $x_{1}{ }^{-}=0$, above the axis $x_{2}{ }^{+}=0$, above the line $l_{7}$ (i.e., $x_{2}{ }^{+}=x_{2 \mathrm{opt}}{ }^{-}$) and at the left of line $l_{8}$ (i.e., $x_{1 \mathrm{opt}}{ }^{+}=x_{1}{ }^{-}$). The maximum value of objective function $F^{-}=50 \cdot x_{1}{ }^{-}-90 \cdot x_{2}{ }^{+}$(i.e., line $\left.l_{6}\right)$ is obtained at the vertex of $A_{2}$, i.e., point $p_{2}(22.23,6.64)$. The optimum lower bound of objective function $F_{\mathrm{opt}}{ }^{-}=514.09$ that can be reached at point $p_{2}$.

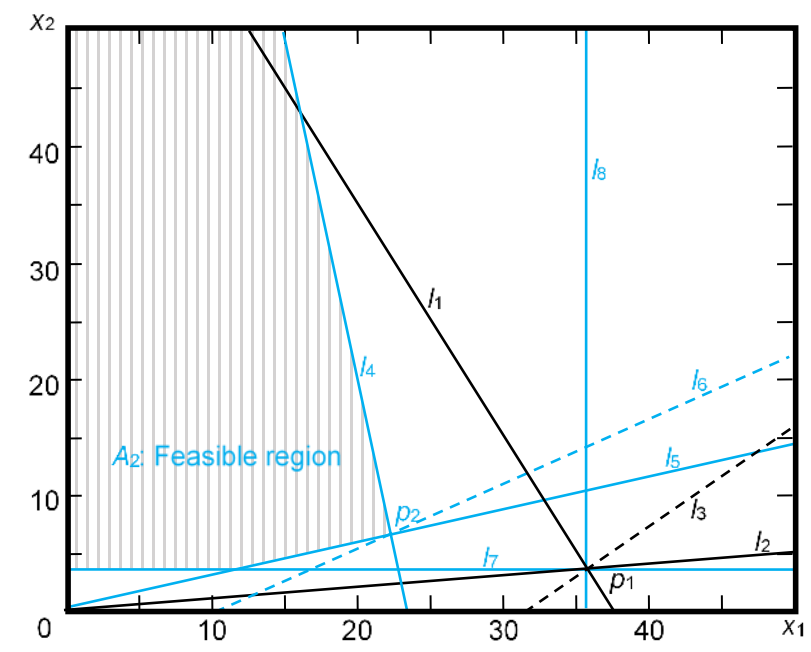

Figure 3. Feasible region and optimum solution of the submodel (14).

As a combination of solutions from models (13) and (14), an initial interval-set solution of the original ILP model (12) is $x_{\text {lopt }}{ }^{ \pm}=[22.23,35.67]$ and $x_{2 \text { opt }}{ }^{ \pm}=[3.67,6.64]$, leading to a rectangular decision space presented as the green grid area $(D)$ in Figure 4. Correspondingly, the maximum value of objective function (12-1) ranges from the maximum objective value of (141) (i.e., 514.09) to that of (13-1) (i.e., 1883.33). This implies that any real value between the optimum objective bounds, i.e., [514.09, 1883.33] for ILP model (12), can be obtained for at least one point in initial decision space $D$. Besides, the recommended solution of any inexact decision variable is a continuous value range in which most of satisfactory decisions are located. Compared to other potential solution formats, e.g., complex formulas and discrete solutions, the interval decision space is more user-friendly and reliable for planners or engineers to guide programming problems especially large-scale ones of thousands of constraints and parameters.

\subsection{Violation Analysis}

Combining feasible regions of models (13) and (14) together, we can have three areas of different feasibilities.

(a) The first one (abbreviated as $R_{1}$ ) is the intersection of two feasible regions $A_{1}$ and $A_{2}$, i.e., $R_{1}=A_{1} \cap A_{2}$. For any element $\boldsymbol{X}\left(x_{1}, x_{2}\right)$ in area $R_{1}$, we can have $\boldsymbol{X} \in A_{1}$ and $\boldsymbol{X} \in A_{2}$, and it is feasible for constraints of both models. Thus, $R_{1}$ is defined as the absolutely feasible region (Huang et al., 1992) of the original ILP model (12). In Figure 4, it is presented as the field filled with grids.

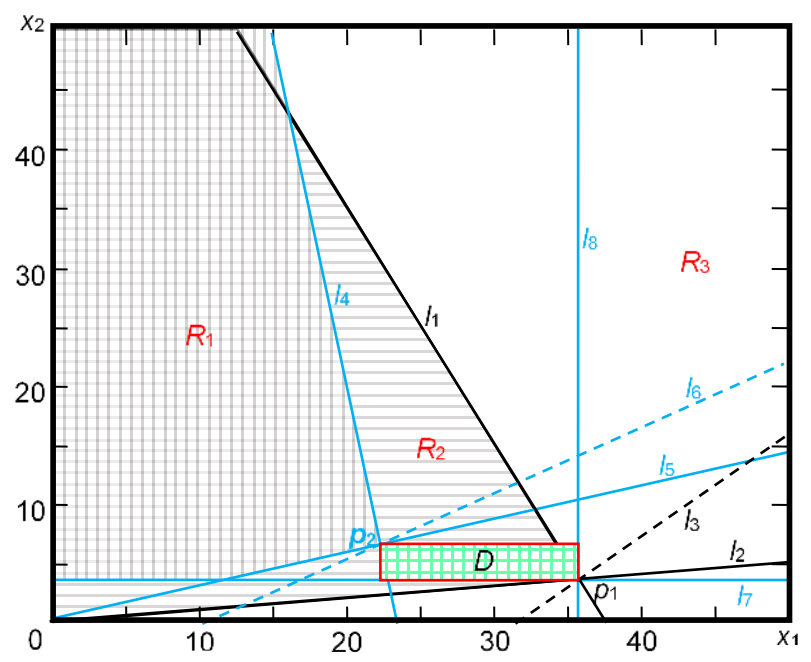

Figure 4. Interval-set solutions of ILP model (12).

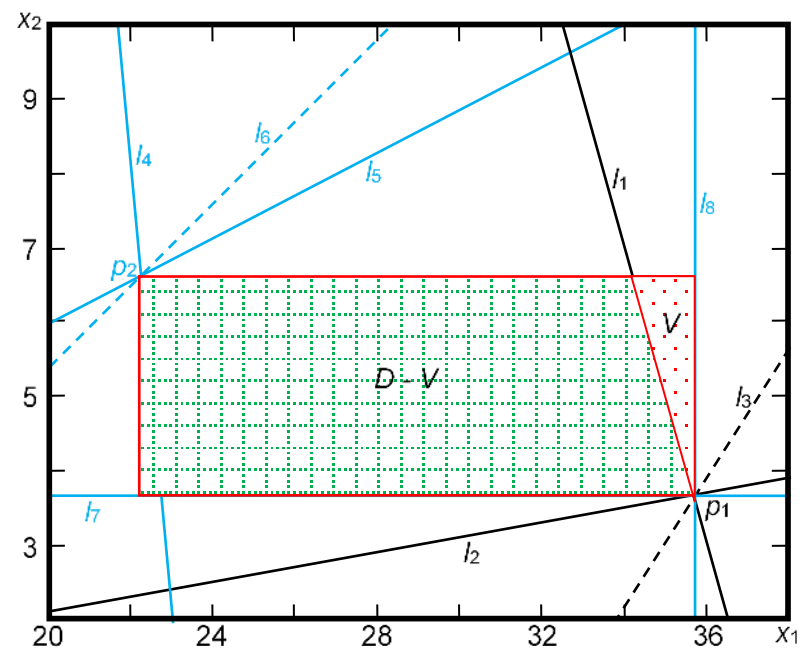

Figure 5. Constraint violation for ILP model (12).

(b) The second one (abbreviated as $R_{2}$ ) is the difference between region $R_{1}$ and the union of two feasible regions $A_{1}$ and $A_{2}$, i.e., $R_{2}=\left(A_{1} \cup A_{2}\right)-R_{1}$. It holds for any element $\boldsymbol{X}\left(x_{1}, x_{2}\right)$ in the area $R_{2}$ that $\boldsymbol{X} \notin R_{1}$ and $X \in A_{1}$ or $A_{2}$. In other words, any element in region $R_{2}$ is feasible for constraints of either model (13) or (14), but not for both models. As defined in (Huang et al., 1992), any solution within $R_{2}$ is softly feasible for ILP model (12). In Figure 4, it is presented as the field filled with parallel lines.

(c) The third one (abbreviated as $R_{3}$ ) is the difference between the two-dimensional real space and the union of two feasible regions $A_{1}$ and $A_{2}$, i.e., $R_{3}=R-\left(A_{1} \cup A_{2}\right)$ where $R=\{X$ $\left(x_{1}, x_{2}\right) \mid x_{j}$ is a real number for $j=1$ and 2$\}$. For any element $(\boldsymbol{X}$ $\left.\left(x_{1}, x_{2}\right)\right)$ in region $R_{3}$, it is infeasible for either model (13) or (14). Therefore, region $R_{3}$ is defined as the absolutely infeasible region (Huang et al., 1992). It is presented as the blank field without any pattern in Figure 4. 
For ILP model (12), any point in the final rectangular decision space $(D)$ obtained from TSA is supposed to be feasible for at least one combination of interval coefficients' whitened values. As presented in Figure 5, however, the top right corner (i.e., red region $V$ ) as a part of initial decision space $D$ is located outside of softly feasible region $R_{2}$. Any point in region $V$ belongs to the absolutely infeasible region $\left(R_{3}\right)$, and violates line $l_{1}$ which corresponds to constraint (13-2) of the first sub-model, i.e., $4 \cdot x_{1}{ }^{+}+2 \cdot x_{2}^{-}=150$.

Given the initial decision space $(D)$ obtained from TSA, the most desired REM scheme for decision makers may correspond to any point in space $D$. If it is located in area $V$, high penalties would be paid for violation of constraint (13-2). Therefore, it is assumed that such violation should be avoided for decision makers who prefer to maximize the net system profit, although its occurrence probability is not significantly high.

As fluctuation of decision variables within bounds $x_{\text {jopt }}{ }^{-}$ and $x_{j \mathrm{opt}}{ }^{+}(j=1,2)$, the maximum values of the left-hand side of constraints (13-2) and (13-3) in the first sub-model are $4 \cdot x_{\text {lopt }}{ }^{+}$ $+2 \cdot x_{2 \mathrm{opt}}{ }^{+}=155.94$, and $x_{1 \mathrm{opt}}{ }^{+}-10 \cdot x_{2 \mathrm{opt}}{ }^{-}=-1$, respectively. Therefore, the first constraint (13-2) is violated. The set of indexes of violated constraints is $\{1\}$ in which only one element is included. The violation range can be obtained from 155.94 $150=5.94$.

\subsection{Contraction of Initial Decision Space}

Referring to the classification method of decision variables in violated constraints that was previously proposed in this study, the second and fourth groups of decision variables are empty, and $x_{1}{ }^{ \pm}$and $x_{2}{ }^{ \pm}$belong to the first and third groups, respectively. Let the contraction ratio $r$ of $x_{2}{ }^{+}$be $0,0.1,0.2,0.3$, $0.4,0.5,0.6,0.7,0.8,0.9$ and 1 , respectively. The third sub-model in the IRLP approach can be formulated as:

$\max F_{*}{ }^{+}=60 \cdot x_{1 *}{ }^{+}-70 \cdot x_{2 *}$

subject to

$4 \cdot\left(x_{1 *^{*}}{ }^{+}-x_{1 \mathrm{opt}}{ }^{+} \cdot r\right)+2 \cdot x_{2 \mathrm{opt}^{+}} \cdot(1-r) \leq 150 \cdot(1-r)$

$x_{1 *}{ }^{+} \in[22.23,35.67]$

$x_{2 *}{ }^{-} \in[3.67,6.64]$

where the upper bound $\left(x_{1^{*}}{ }^{+}\right)$of $x_{1 \text { opt }}{ }^{ \pm}$and the lower bound $\left(x_{2^{*}}{ }^{-}\right)$ of $x_{2 \mathrm{opt}}{ }^{ \pm}$are bounds of decision variables that should be adjustted. The optimal solutions of linear programming model (15) corresponding to varied constraint contraction ratio $r$ can be obtained from the simplex method. They are presented in table 1.

On the basis of solutions from the third sub-model, the fourth sub-model can be formulated as:

$\min F^{*}{ }^{-}=50 \cdot x_{1^{*}}{ }^{-}-90 \cdot x_{2 *^{+}}$

subject to

$4 \cdot x_{1 \mathrm{opt}}{ }^{+} \cdot r+2 \cdot\left(x_{2 *^{*}}{ }^{+}-x_{2 \mathrm{opt}}{ }^{+} \cdot(1-r)\right) \leq 150 \cdot r$

$x_{1 \mathrm{opt}}{ }^{-} \leq x_{1 *}{ }^{-} \leq x_{1 \mathrm{opt} *^{+}}$ $x_{2 \mathrm{opt}}{ }^{+} \geq x_{2 *^{+}} \geq x_{2 \mathrm{opt}^{*}}$

where the lower bound $x_{1 *^{-}}$of $x_{1 \mathrm{opt}}{ }^{ \pm}$and the upper bound $x_{2 *^{+}}$of $x_{2 \text { opt }}{ }^{ \pm}$are bounds of decision variables that should be adjusted. The simplex method is employed again for solving linear programming model (16). The optimal solutions corresponding to varied contraction ratios are presented in Table 1. Combining solutions from the third and fourth sub-models together, we can have the final interval-set solutions under varied contraction ratios as presented in Figure 6. It is indicated that the proposed $I R L P$ approach enables achieving the maximal decision space in which infeasible solutions of sub-model ILPI are excluded through sub-models ILP3 and ILP4.

Table 1. Solutions of sub-models (15) and (16)

\begin{tabular}{lllllll}
\hline$r$ & \multicolumn{3}{c}{ Solution of model (15) } & \multicolumn{3}{c}{ Solution of model (16) } \\
\cline { 2 - 7 } & $x_{\text {lopt* }^{*}}$ & $x_{2 \text { opt* }^{*}}$ & $F_{\text {opt* }^{*}}$ & $x_{1 \text { opt* }^{-}}$ & $x_{2 \text { opt* }^{*}}$ & $F_{\text {opt* }^{*}}$ \\
\hline 0 & 34.18 & 3.67 & 1794.24 & 22.23 & 6.64 & 514.09 \\
0.1 & 34.33 & 3.67 & 1803.15 & 22.23 & 6.34 & 540.82 \\
0.2 & 34.48 & 3.67 & 1812.06 & 22.23 & 6.04 & 567.55 \\
0.3 & 34.63 & 3.67 & 1820.97 & 22.23 & 5.75 & 594.27 \\
0.4 & 34.78 & 3.67 & 1829.88 & 22.23 & 5.45 & 621.00 \\
0.5 & 34.92 & 3.67 & 1838.79 & 22.23 & 5.15 & 647.73 \\
0.6 & 35.07 & 3.67 & 1847.70 & 22.23 & 4.86 & 674.46 \\
0.7 & 35.22 & 3.67 & 1856.61 & 22.23 & 4.56 & 701.18 \\
0.8 & 35.37 & 3.67 & 1865.52 & 22.23 & 4.26 & 727.91 \\
0.9 & 35.52 & 3.67 & 1874.42 & 22.23 & 3.96 & 754.64 \\
1 & 35.67 & 3.67 & 1883.33 & 22.23 & 3.67 & 781.36 \\
\hline
\end{tabular}

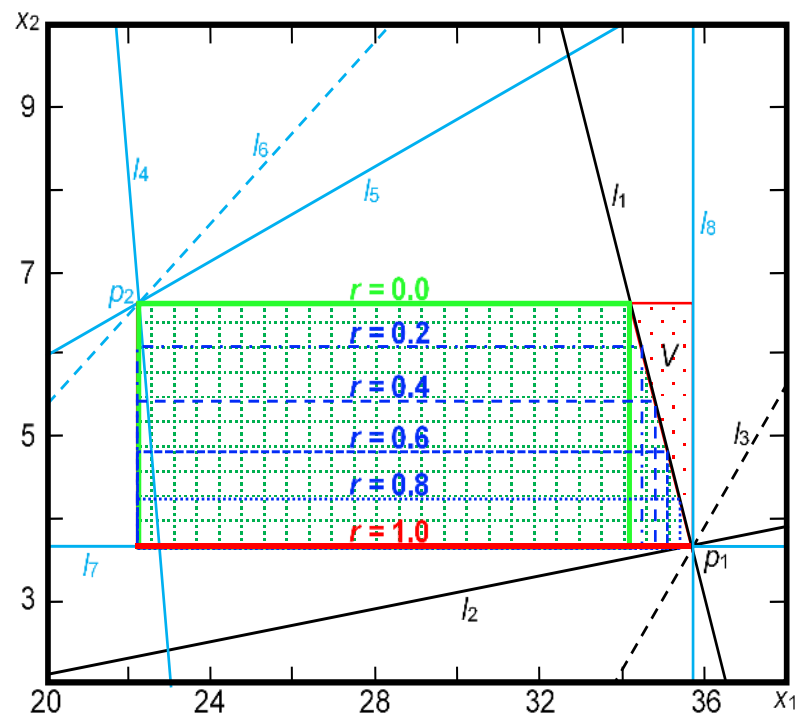

Figure 6. Solutions of ILP model (12) through the IRLP approach.

\subsection{Post-optimality Analysis}

Optimistic decision makers may prefer to high system profits that can be accomplished through setting contraction ratio as 1 . Accordingly, the optimal decision ranges $x_{1 \mathrm{opt}^{*}}{ }^{ \pm}=[22.23$, 
$35.67]$ and $x_{2 \mathrm{opt}^{*}{ }^{ \pm}}=3.67$. The maximum value range of objecttive function is $F_{\text {opt* }^{*}}=[781.36,1883.33]$. For conservative decision makers who expect low system profit and low constraint violation risk under uncertainties, the preferable solutions are corresponded by lowest contraction ratios. In detail, the optimal decision ranges $x_{1 \mathrm{opt}^{*}}{ }^{ \pm}=[22.23,34.18]$ and $x_{2 \mathrm{opt}^{*}}{ }^{ \pm}=[3.67$ 6.64]. The maximum objective value range is $F_{\text {opt* } *^{ \pm}}=[514.09$, 1794.24]. These solutions are also of high priority for decision makers who desire a large decision space, if the size of decision space is quantified by the proposed index DSR. As for other decision makers, they can be provided with interval-set solutions based on analysis of the correspondence between their preferences and constraint contraction ratios.

\section{Discussions}

\subsection{Connection with TSA Method}

The proposed IRLP approach can be taken as a supplement to the TSA method developed by Huang et al. (1992). The latter one is incorporated into the former one for initializing a decision space through sub-models ILPI and ILP2. Effectiveness of TSA at obtaining a continuous decision space is remained in IRLP. To mitigate constraint-violation problems of TSA, the cause of violation is revealed in the section of violation analysis based on the definition of violation criterion (i.e., constraints of sub-model ILPI of which violation may lead to high penalties to real-world $I L P$ systems). Violation ranges of constraints are quantified, leading to proposition of contraction ranges and contraction ratios. A series of contraction ratios are provided by decision makers of diverse preferences for violated constraints of sub-model ILPI. As a recourse measure for constraint violation, the initial decision space is contracted through sub-models ILP3 and ILP4 that correspond to optimistic solutions and conservative ones, respectively. A continuous decision space is finalized by combining crisp solutions of two linear programming sub-models. It is located within the initial decision space while solutions causing violation of constraints of sub-model ILP1 are removed. Contraction ratios can help distinguish preferences of decision makers, e.g., low values for ones who pursue conservative schemes or a large decision space. Combining solutions under all combinations of contraction ratios for violated constraints can facilitate decision makers identifying the maximal decision space which is the initial one excluding infeasible schemes. Therefore, the proposed IRLP approach enables mitigation of constraint-violation problems while remainning strengthens of TSA and adapting to decision makers of diverse preferences. The reliability of the optimization process for ILP problems is enhanced in comparison with the TSA method.

\subsection{Comparison with Improved ILP Methods}

The proposed IRLP approach is not the first one to examine constraint-violation problems of the TSA method. Previously, a modified interval linear programming (MILP) method, a three-step $(T h S)$ method and a robust two-step (RTS) method were contributed by Zhou et al. (2008), Huang and Cao (2011) and Fan and Huang (2012), respectively. In MILP, an additional constraint is coupled with constraints of sub-model ILP2 for avoiding violation of constraints. As for ILP model (12), the additional constraint of sub-model (14) can be formulated as $x_{2}{ }^{+}-2 \cdot 3.67 \leq 0$. The optimal solution of TSA is $x_{1 \mathrm{opt}}{ }^{ \pm}=[22.23$, $35.67]$ and $x_{2 \mathrm{opt}}{ }^{ \pm}=[3.67,6.64]$. It is identical with that of MILP, implying that constraint violation remains for MILP. In the ThS method, another sub-model is constructed to constrict the initial decision space obtained from first two sub-models ILPI and ILP2. Parameters constricting ratios of decision variables are introduced to reflect constricting ranges of initialized intervalset solutions. They are optimized through the third sub-model subject to avoidance of constraint violation. However, the submodel may be a nonlinear programming model which is of limited applicability to large-scale $I L P$ problems. Otherwise, it may lead to a great waste of the initial decision space in the process of constricting initialized solutions synchronously. In the RTS method, sub-model ILP2 that focuses on conservative schemes is solved prior to sub-model ILP1. A group of additional constraints is incorporated into the original sub-model ILPI for avoiding occurrence of constraint violation. However, RTS is challenged by possible sacrifice of optimistic solutions that correspond to high system profits. RTS is only adaptive for conservative decision makers who concern schemes of relatively low risks. In contrast, the IRLP approach can help decision makers of various preferences identify desired schemes while demands of conservative ones can be satisfied. The applicable scope of the RTS method is just a particular case of the IRLP approach. Moreover, all existing improved ILP methods cannot provide the largest decision space which does not include solutions that may cause constraint violation. Part of decision alternatives are neglected due to ineffectiveness of these methods. This limitation is overcame by the IRLP approach, which is helpful for enhancing reliability of the decision support for $R E M$ problems under interval uncertainties.

\subsection{Comparison with Discrete ILP Methods}

In addition to aforementioned methods (e.g., MILP, ThS and $R T S$ ), multiple efforts are made to solving of ILP models. For instance, Tong (1994), Levin (1994) and Chi-nneck and Ramadan (2000) proposed methods that could obtain the most optimistic decision scheme (denoted as $\boldsymbol{X}_{u}$ ) of ILP model (1) from a best-case sub-model as well as the most conservative decision scheme (denoted as $\boldsymbol{X}_{l}$ ) from a worst-case sub-model. Let these methods be named as discrete interval linear programming and be abbreviated as DILP in this study. These methods only focus on two extreme cases corresponding to particular combinations of inexact coefficients in ILP model (1). If at least one optimal solution exists for the best-case sub-model, it can be deduced from (Tong, 1994) and (Huang et al., 1992) that there would be infinite possibly optimal solutions corresponding to infinite combinations of coefficients. Either $\boldsymbol{X}_{u}$ or $\boldsymbol{X}_{l}$ is only a particular case out of infinite ones. Almost all of decision alternatives including the possibly most desired decision scheme that may be located between the two extreme cases are neglected due to discreteness of the decision space in DILP methods. Furthermore, constraints may be violated for DILP methods under the assumption of continuity of decision space. Let 
the value of objective function (1-1) corresponding to $\boldsymbol{X}_{u}$ and $\boldsymbol{X}_{l}$ be denoted as $F_{\text {uopt }}$ and $F_{\text {lopt }}$, respectively. The decision space (denoted as DILPDS) constrained by the two most extreme schemes (i.e., $\boldsymbol{X}_{u}$ or $\boldsymbol{X}_{l}$ ) may involve solutions that are infeasible for all combinations of inexact coefficients. Let the set of these solutions be denoted as DILPInF. Any element in DILPInF, especially ones around $\boldsymbol{X}_{u}$ or $\boldsymbol{X}_{l}$, may lead to extremely high penalties for infeasibility, and needs to be removed from DILPDS. In comparison, these challenges are effectively mitigated in the proposed IRLP approach.

\subsection{Comparison with Robust Linear Programming}

The IRLP approach is developed for solving ILP models and guiding $I L P$ problems. Management schemes can also be provided by robust linear programming $(R L P)$ methods. The $I L P$ models are equivalent with $R L P$ problems in particular cases. An $R L P$ model corresponding to ILP model (1) can be formulated as Max $\{\boldsymbol{C X} \mid \boldsymbol{A X} \leq \boldsymbol{b} ; \boldsymbol{X} \geq \mathbf{0}\}$ where $\boldsymbol{X}$ is a $n \times 1$ vector of non-negative decision variables $x_{j}(j=1,2, \ldots, n) ; \mathbf{0}=\{0\}^{n \times 1}$; $\mathbf{C}=\left\{c_{j}\right\}^{1 \times n}, \mathbf{A}=\left\{a_{i j}\right\}^{m \times n}$, and $\boldsymbol{b}=\left\{b_{i}\right\}^{m \times 1}$; positive integers $n$ and $m$ are numbers of decision variables $\left\{x_{j}\right\}$ and constraints, respectively; any vector or matrix of coefficients (i.e., $\boldsymbol{C}, \boldsymbol{A}$, or $\boldsymbol{b})$ belongs to an uncertainty set $\boldsymbol{U}$ where $\boldsymbol{U}=\left\{\boldsymbol{C}=\left\{c_{j}\right\}^{1 \times n} \mid c_{j}{ }^{-}\right.$ $\left.\leq c_{j} \leq c_{j}^{+} ; j=1,2, \ldots, n\right\} \cup\left\{\boldsymbol{A}=\left\{a_{i j}\right\}^{m \times n} \mid a_{i j}{ }^{-} \leq a_{i j} \leq a_{i j}{ }^{+} ; i=1\right.$, $2, \ldots, m ; j=1,2, \ldots, n\} \cup\left\{\boldsymbol{b}=\left\{b_{i}\right\}^{m \times 1} \mid b_{i}{ }^{-} \leq b_{i} \leq b_{i}{ }^{+} ; i=1,2\right.$, $\ldots, m\}$. The appeal of robust linear programming is that its solutions and performances remain relatively unchanged when exposed to uncertain conditions (Beyer and Sendhoff, 2007). A robust solution of ILP model (1) can be obtained from a robust counterpart of the $R L P$ model (abbreviated as $R C L P$ ). The model can be expressed as Max $\{f \mid F \leq \boldsymbol{C X} ; \boldsymbol{A} \boldsymbol{X} \leq \boldsymbol{b} ; \boldsymbol{X} \geq \mathbf{0} ; \forall \boldsymbol{A} \subseteq$ $\boldsymbol{U} ; \forall \boldsymbol{C} \subseteq \boldsymbol{U} ; \forall \boldsymbol{b} \subseteq \boldsymbol{U}\}$. As stated in (Beyer and Sendhoff, 20$07)$, however, the robust solution obtained from the RCLP model is the most conservative decision scheme. Higher system profits are sacrificed for pursuing higher robustness of solutions, which cannot be acceptable for all decision makers of diverse preferences in real-world programming problems, at least for optimistic ones who are willing to chase high profits while taking risks of solution infeasibility. In contrast, the IRLP approach for $I L P$ models is of higher adaptability and enables provision of a continuous decision space for decision making under interval uncertainties.

\subsection{Potential Extensions}

Although the proposed IRLP approach effectively mitigates constraint violation problems in solving $I L P$ models, it is of multiple limitations due to complexities of real-world resources and environmental management systems. For instance, contraction ratios are introduced to reflect preferences of decision makers, and they should be determined prior to contraction of the initial decision space. There is still a lack of scientific tool to help decision makers identify the desired values of these ratios. Elaboration of penalties of constraint violation may be helpful for achieving it. In this study, the criterion of constraint violation is assumed to be constraints of sub-model ILPI due to relatively high penalties. Effectiveness of the IRLP approach relies on the assumption. As a result, it is still possible that the obtained solutions lead to violation of constraints under other criteria such as constraints of sub-model ILP2. In these cases, the IRLP approach should be corrected to reflect realities of real-world $I L P$ problems. The IRLP approach is dependent with assumptions 1 to 6 that hold for most resources and environmental management problems. Nonetheless, it is possible that these assumptions do not hold for some cases. Corresponding revisions are desired for avoiding ineffectiveness of the IRLP approach. The suggested solution of the IRLP approach is a series of interval sets. It provides a decision space of which any solution may be the desired scheme for decision makers. However, the feasibility and optimality of any solution is connected with whitened values of interval-set coefficients in ILP models. To facilitate decision makers identifying the most desired scheme, analysis of the correspondence between them may be demanded from the viewpoint of applicability. In addition, there may be a number of other complexities in ILP systems, e.g., multiple objectives, nonlinear relationships, discrete alternatives, parameter interactions, subjective information, random disturbances, and joint uncertainties. Coupling IRLP with other optimization methods deserves an attempt to reflect these complexities and provide reliable decision support for engineering practices. Therefore, a lot of efforts will be made in the following studies to overcome these challenges, enlarge adaptability of the IRLP approach, enhance reliability of decision support processes, and promote effective management of resources and environmental systems.

\section{Conclusions}

An IRLP approach was proposed in this study. Based on a review of ILP models, the TSA approach and their significances to REM problems, sub-models ILPI and ILP2 in TSA were employed to initialize a decision space for IRLP. Causes of constraint violation were examined based on identification of a violation criterion. Contraction ratios were defined after revelation of violation ranges of constraints. As a recourse measure to constraint violation problems, two linear programming sub-models (ILP3 and ILP4) were constructed given a series of contraction ratios. A hypercube decision space where infeasible solutions were excluded through sub-models ILP3 and ILP4 was obtainned for decision makers. Post-optimality analysis was conducted to deal with barriers in applying the IRLP approach to realworld ILP problems. An ILP problem was introduced to demonstrate procedures and effectiveness of the IRLP approach. Strengthens and shortcomings of the IRLP approach were revealed from comparisons with existing ILP methods.

Results indicated that the IRLP approach was effective at resolving the constraint-violation problem of the TSA approa$\mathrm{ch}$, reproducing the largest decision space which did not include infeasible solutions and enhancing reliability of decision support for REM. A hypercube decision space that covered most alternative schemes could be provided for facilitating REM under interval uncertainties. Demands of decision makers of diverse preferences could be satisfied by adjusting values of contraction ratios, e.g., low values for ones who pursued conserva- 
tive schemes or a large decision space. The reliability of the optimization process for $I L P$ problems was enhanced in compareson with existing ILP methods.

Complexities of real-world $R E M$ problems may require further improvements of the $I R L P$ approach in the future studies. For instance, elaboration of penalties of constraint violation is helpful for identifying the desired values of contraction ratios. The $I R L P$ approach should be corrected to adapt to various constraint-violation criteria. Corresponding revisions are desired for avoiding ineffectiveness of the IRLP approach when related assumptions do not hold. To facilitate decision makers identifying the most desired scheme, analysis of the correspondence between solutions and coefficient combinations may be demanded from the viewpoint of applicability. Coupling IRLP with other optimization methods deserves an attempt to reflect diverse complexities (e.g., multiple objectives, nonlinear relationships, discrete alternatives, parameter interactions, subjecttive information, random disturbances, and joint uncertainties) and provide reliable decision support for $R E M$.

Acknowledgments: This research was supported by the National Key Research and Development Plan (2016YFA0601502, 2016YFC0502800 ), the Program for Innovative Research Team in University (IRT1127), the 111 Project (B14008) and the Natural Science and Engineering Research Council of Canada.

\section{References}

Ben-Tal, A., and Nemirovski, A. (2002).Robust optimization methodology and applications. Math.Program., 92(3), 453-480. http://dx. doi.org/10.1007/s101070100286

Ben-Tal, A., El Ghaoui, L., and Nemirovski, A. (2009). Robust Optimization. Princeton Series in Applied Mathematics, Princeton University Press, 9-16. http://dx.doi.org/10.1515/9781400831050

Bertsimas, D., and Sim, M. (2004). The Price of Robustness. Oper. Res., 52 (1): 35-53. http://dx.doi.org/10.1287/opre.1030.0065

Bertsimas, D., Brown, D.B., and Caramanis, C. (2011). Theory and applications of robust optimization. SIAM rev., 53(3), 464-501. http: //dx.doi.org/10.1137/080734510

Beyer, H.G., and Sendhoff, B. (2007). Robust optimization - a comprehensive survey.Comput. Methods Appl. Mech. Eng., 196(33), 31903218. http://dx.doi.org/10.1016/j.cma.2007.03.003

Cai, Y.P., Huang, G.H., Yang, Z.F., Lin, Q.G., and Tan, Q. (2009). Community-scale renewable energy systems planning under uncertainty - An interval chance-constrained programming approach. Renew. Sustain. Energy Rev., 13(4), 721-735. http://dx.doi.org/10.1016/j.rse r.2008.01.008

Cai, Y.P., Huang, G.H., Yang, Z.F., and Tan, Q. (2009). Identification of optimal strategies for energy management systems planning under multiple uncertainties. Applied Energy, 86(4), 480-495. https: //doi.org/10.1016/j.apenergy.2008.09.025

Chanas, S., and Kuchta, D. (1996). Multiobjetive programming in optimization of interval objective functions - A generalized approach. Eur. J. Oper. Res., 94, 594-598. http://dx.doi.org/10.1016/0377-221 7(95)00055-0

Chang, N., Chen, H., Shaw, D., and Yang, C. (1997). Water Pollution Control in River Basin by Interactive Fuzzy Interval Multiobjective Programming. J. Environ. Eng., 123(12), 1208-1216. http://dx.doi.o rg/10.1061/(ASCE)0733-9372(1997)123:12(1208)

Chang, N.B., Chen, Y.L., and Wang, S.F. (1997). A fuzzy interval multiobjective mixed integer programming approach for the optimal planning of solid waste management systems. Fuzzy Sets Syst., 89(1),
35-60. http://dx.doi.org/10.1016/S0165-0114(96)00086-3

Charnes, A., Granot, F., and Phillips, F. (1977). An algorithm for solving interval linear programming problems. Oper. Res., 25, 688-69 5. http://dx.doi.org/10.1287/opre.25.4.688

Chen, C., Li, Y.P., and Huang, G.H. (2013). An inexact robust optimization method for supporting carbon dioxide emissions management in regional electric-power systems. Energy Econ., 40, 441-45 6. http://dx.doi.org/10.1016/j.eneco.2013.07.022

Chen, X.J., Huang, G.H., An, C.J., Yao, Y., and Zhao, S. (2018). Emerging $\mathrm{N}$-nitrosamines and $\mathrm{N}$-nitramines from amine-based postcombustion CO2 capture - A review. Chem. Eng. J., 335, 921-935. https://doi.org/10.1016/j.cej.2017.11.032

Cheng, G.H., Huang, G.H., Dong, C., Xu, Y., Chen, J.P., Chen, X.J., and Li, K.L. (2017). Distributed mixed-integer fuzzy hierarchical programming for municipal solid waste management. Part II: scheme analysis and mechanism revelation. Environ. Sci. Pollut. Res., 24(9), 8711-8721. https://doi.org/10.1007/s11356-017-8574-8

Cheng, G.H., Huang, G.H., Dong, C., Xu, Y., Chen, X.J., and Chen, J.P. (2017). Distributed mixed-integer fuzzy hierarchical programming for municipal solid waste management. Part I: System identification and methodology development. Environ. Sci. Pollut. Res., 24(8), 7236-7252. https://doi.org/10.1007/s11356-016-8284-7

Cheng, G.H., Huang, G.H., and Dong, C. (2017). Convex contractive interval linear programming for resources and environmental systems management. Stoch. Environ. Res. Risk Assess., 31(1), 205-22 4. https://doi.org/10.1007/s00477-015-1187-1

Cheng, G.H., Huang, G.H., and Dong, C. (2015). Synchronic interval Gaussian mixed-integer programming for air quality management. Sci. Total Environ., 538, 986-996. https://doi.org/10.1016/j.scitoten v.2015.08.099

Cheng, G.H., Huang, G.H., Li, Y.P., Cao, M.F., and Fan, Y.R. (2009). Planning of municipal solid waste management systems under dual uncertainties: a hybrid interval stochastic programming approach. Stoch. Environ. Res. Risk Assess., 23(6), 707. https://doi.org/10.100 7/s00477-008-0251-5

Chinneck, J.W., and Ramadan, K. (2000). Linear programming with interval coefficients. J. Oper. Res. Soc., 51(2), 209-220. http://dx.do i.org/10.1057/palgrave.jors.2600891

Dantzig, G.B. (1963). Linear Programming and Extensions. Princeton University Press, Princeton, New Jersey.

Dong, C., Huang, G.H., and Tan, Q. (2015). A robust optimization modelling approach for managing water and farmland use between anthropogenic modification and ecosystems protection under uncertainties. Ecol. Eng., 76, 95-109. https://doi.org/10.1016/j.ecoleng. 2014.04.003

Dong, C., Huang, G.H., Cai, Y.P., and Liu, Y. (2013). Robust planning of energy management systems with environmental and constraintconservative considerations under multiple uncertainties. Energy Convers. Manag., 65, 471-486. https://doi.org/10.1016/j.enconman. 2012.09.001

Dong, C., Huang, G.H., Cai, Y.P., and Xu, Y. (2011). An interval-parameter minimax regret programming approach for power management systems planning under uncertainty. Appl. Energ., 88(8), 28352845. https://doi.org/10.1016/j.apenergy.2011.01.056

Dong, C., Huang, G.H., Cai, Y.P., Cheng, G.H., and Tan, Q. (2016). Bayesian interval robust optimization for sustainable energy system planning in Qiqihar City, China. Energ. Econ., 60, 357-376. https:// doi.org/10.1016/j.eneco.2016.10.012

Dong, C., Huang, G.H., Cai, Y.P., Li, W., and Cheng, G.H. (2014a). Fuzzy interval programming for energy and environmental systems management under constraint-violation and energy-substitution effects: a case study for the city of Beijing. Energ. Econ., 46, 375394. https://doi.org/10.1016/j.eneco.2014.09.024

Dong, C., Huang, G.H., Cai, Y.P., Yue, W.C., and Rong, Q.Q. (2014b). An interval-parameter fuzzy linear programming approach for accounting and planning of energy-environmental management 
systems. J. Environ. Account. Manag., 2(1):13-29. https://doi.org/ 10.5890/JEAM.2014.03.002

Dong, C., Huang, G. H., Tan, Q., and Cai, Y.P. (2014c). Coupled planning of water resources and agricultural landuse based on an inexact-stochastic programming model. Front. Earth Sci., 8(1), 70-80. https://doi.org/10.1007/s11707-013-0388-5

Dong, C., Huang, G.H., Cai, Y.P., and Liu, Y. (2012). An inexact optimization modeling approach for supporting energy systems planning and air pollution mitigation in Beijing City. Energy, 37(1), 673688. http://dx.doi.org/10.1016/j.energy.2011.10.030

Dong, C., Tan, Q., Huang, G.H., and Cai, Y.P. (2014d). A dual-inexact fuzzy stochastic model for water resources management and nonpoint source pollution mitigation under multiple uncertainties. $H y$ drol. Earth Sys. Sci., 18(5), 1793-1803. https://doi.org/10.5194/ hess $-18-1793-2014$

Fan, Y.R., and Huang, G.H. (2012). A robust two-step method for solving interval linear programming problems within an environmental management context. J. Environ. Inf., 19(1), 1-9. http://dx.doi.org/1 $0.3808 /$ jei.201200203

Gabrel, V., Murat, C., and Thiele, A. (2014). Recent advances in robust optimization: An overview. Eur. J. Oper. Res., 235(3), 471-483. http ://dx.doi.org/10.1016/j.ejor.2013.09.036

Guo, H.C., Liu, L., and Huang, G.H. (2003). A stochastic water quality forecasting system for the Yiluo River. J. Environ. Inf., 1(2), 18-32. http://dx.doi.org/10.3808/jei.200300010

Han, J.C., Huang, G.H., Zhang, H., and Li, Z. (2013). Optimal land use management for soil erosion control by using an interval-parameter fuzzy two-stage stochastic programming approach. Environ. Manage., 52(3), 621-638. http://dx.doi.org/10.1007/s00267-013-01 22-9

Hladık, M. (2012). Interval linear programming: A survey. Linear program.new front. theory appl., 85-120.

Hu, Q., Huang, G.H., Cai, Y.P. and Sun, W. (2014). Planning of electric power generation systems under multiple uncertainties and constraint-violation levels. J. Environ. Inf., 23(1), 55-64. http://dx. doi:1 $0.3808 /$ jei.201400257

Hu, M., Huang, G.H., Sun, W., and Li, Y.P. (2013). Inexact quadratic joint-probabilistic programming for water quality management under uncertainty in the Xiangxi River, China. Stochastic Environ. Re s. Risk Assess., 27(5), 1115-1132. http://dx.doi.org/10.1007/s00477012-0648-Z

Huang, G.H., and Cao, M.F. (2011). Analysis of solution methods for interval linear programming. J. Environ. Inf., 17(2), 54-64. http://dx. doi.org/10.3808/jei.201100187

Huang, G.H., Baetz, B.W., and Patry, G.G. (1992). A grey linear programming approach for municipal solid waste management planning under uncertainty. Civ. Eng. Environ. Syst., 9(4), 319-335. http: //dx.doi.org/10.1080/02630259208970657

Huang, G.H., Baetz, B.W., and Patry, G.G. (1992). An interval linear programming approach for municipal solid waste management planning under uncertainty. Civ. Eng. Syst., 9, 319-335. http://dx.doi.or $\mathrm{g} / 10.1080 / 02630259208970657$

Huang, G.H., Chi, G.F., and Li, Y.P. (2005a). Long-term planning of an integrated solid waste management system under uncertainty - I. model development. Environ. Eng. Sci., 22(6), 823-834. http://dx. doi.org/10.1089/ees.2005.22.823

Huang, G.H., Chi, G.F., and Li, Y.P. (2005b). Long-term planning of an integrated solid waste management system under uncertainty II.A North American case study. Environ. Eng. Sci., 22(6), 835-853. http://dx.doi.org/10.1089/ees.2005.22.835

Huang, G.H., Cohen, S.J., Yin, Y.Y., and Bass, B. (1998). Land resources adaptation planning under changing climate - a study for the Mackenzie Basin. Resour. Conserv. Recycling, 24(2), 95-119. http:// dx.doi.org/10.1016/S0921-3449(98)00044-5

Inuiguchi, M., and Sakawa, M. (1995). Minimax regret solution to linear programming problems with an interval objective function. $E u-$ r. J. Oper. Res., 86, 526-536. http://dx.doi.org/10.1016/0377-2217(9
4)00092-Q

Inuiguchi, M., and Sakawa, M. (1997). An achievement rate approach to linear programming problems with an interval objective function. J. Oper. Res. Soc., 48(1), 25-33. http://dx.doi.org/10.1057/palgrave. jors. 2600322

Inuiguchi, M., Ramik, J., Tanino, T., and Vlach, M. (2003). Satisficing solutions and duality in interval and fuzzy linear programming. Fuzzy Sets Syst., 135, 151-177. http://dx.doi.org/10.1016/S0165-0114 (02)00253-1

Ishibuchi, H., and Tanaka, H. (1990). Multiobjective programming in optimization of the interval objective function. Eur. J. Oper. Res., 48, 219-225. http://dx.doi.org/10.1016/0377-2217(90)90375-L

Jaccard, J. (1998). Interaction effects in factorial analysis of variance (No. 118). Sage.

Jin, J.L., Wang, S.J., and Wei, Y.M. (2004). Ideal Interval Method Based Model for Water Quality Evaluation. Nat. Sci., 2(1), 24-28.

Levin, V.I. (1994). Interval discrete programming. Cybernetics Syst. Anal., 30(6), 866-874. http://dx.doi.org/10.1007/BF02366445

Li, G.C., Huang, G.H., Sun, W., and Ding, X.W. (2014). An inexact optimization model for energy-environment systems management in the mixed fuzzy, dual-interval and stochastic environment. $R e$ new. Energy, 64, 153-163. http://dx.doi.org/10.1016/j.renene.2013.1 1.013

Li, M.W., Li, Y.P., and Huang, G.H. (2011). An interval-fuzzy two-stage stochastic programming model for planning carbon dioxide trading under uncertainty. Energy, 36(9), 5677-5689. http://dx.doi.org/ 10.1016/j.energy.2011.06.058

Li, Y.P., and Huang, G.H. (2009). Fuzzy-stochastic-based violation analysis method for planning water resources management systems with uncertain information. Inf. Sci., 179(24), 4261-4276. https://do i.org/10.1016/j.ins.2009.09.001

Li, Y.P., Huang, G.H., Huang, Y.F., and Zhou, H.D. (2009). A multistage fuzzy-stochastic programming model for supporting sustainnable water resources allocation and management. Environ. Model. Software, 24(7), 786-797. https://doi.org/10. 1016/j.envsoft.2008. 11.008

Linkov, I., Satterstrom, F.K., Kiker, G., Batchelor, C., Bridges, T., and Ferguson, E. (2006). From comparative risk assessment to multicriteria decision analysis and adaptive management: Recent developments and applications. Environ. Int., 32(8), 1072-1093. http://dx. doi.org/10.1016/j.envint.2006.06.013

Lv, Y., Huang, G.H., Li, Y.P., Yang, Z.F., Liu, Y., and Cheng, G.H. (2010). Planning regional water resources system using an interval fuzzy bi-level programming method. J. Environ. Inf., 16(2), 43-56. http://dx.doi.org/10.3808/jei.201000177

Maqsood, I., and Huang, G.H. (2003). A two-stage interval-stochastic programming model for waste management under uncertainty. J. Air Waste Manage. Assoc., 53(5), 540-552. http://dx.doi.org/ 10.108 0/10473289.2003.10466195

Maqsood, I., Huang, G.H., and Yeomans, J.S. (2005). An interval-parameter fuzzy two-stage stochastic program for water resources management under uncertainty. Eur. J. Oper. Res., 167(1), 208-225. http://dx.doi.org/10.1016/j.ejor.2003.08.068

Moore, R.E. (1979). Method and application of interval analysis. SIAM, Philadelphia. http://dx.doi.org/10.1137/1.9781611970906

Nasiri, F., and Huang, G.H. (2007). Ecological viability assessment: A fuzzy multiple-attribute analysis with respect to three classes of ordering techniques. Ecol. Inf., 2(2), 128-137. http://dx.doi.org/10.10 16/j.ecoinf.2007.05.003

Nasiri, F., Manuilova, A., and Huang, G.H. (2009). Environmental policy analysis in freight transportation planning: An optimality assessment approach. Int. J. Sustain. Transp., 3(2), 88-109. http://dx.doi. org/10.1080/15568310701779519

Ni, J.R., Wu, A., Li, T.H., Yue, Y. and Borthwick, A.G.L. (2014). Efficient Soil Loss Assessment for Large Basins Using Smart Coded Polygons. J. Environ. Inf., 23(2), 47-57. http://dx. doi:10.3808/jei. 


\section{4}

Nikoo, M.R., Kerachian, R., and Karimi, A. (2012). A Nonlinear Interval Model for Water and Waste Load Allocation in River Basins. Water Resour. Manage., 26(10), 2911-2926. http://dx.doi.org/10.10 07/s11269-012-0056-7

Nikoo, M.R., Kerachian, R., Poorsepahy-Samian, H. (2012). An Interval Parameter Model for Cooperative Inter-Basin Water Resources Allocation Considering the Water Quality Issues. Water Resour. Manage., 26(11), 3329-3343. http://dx.doi.org/10.1007/s11269-012-00 74-5

Niu, X.Z., Williams, J. Miller Z., Lehnert, D.K., Bills, B. and Brantley, S.L. (2014). An Ontology Driven Relational Geochemical Database for the Earth's Critical Zone: CZchemDB. J. Environ. Inf., 23(2), 10-23. http://dx. doi:10.3808/jei.20140025

Pires, A., Chang, N.B., and Martinho, G. (2011). An AHP-based fuzzy interval TOPSIS assessment for sustainable expansion of the solid waste management system in Setúbal Peninsula, Portugal. Resour. Conserv. Recycling, 56(1), 7-21. http://dx.doi.org/10.1016/j.resconr ec.2011.08.004

Saltelli, A., and Bolado, R. (1998). An alternative way to compute Fourier amplitude sensitivity test (FAST). Comput. Stat. Data Anal., 26(4), 445-460. http://dx.doi.org/10.1016/S0167-9473(97)00043-1

Sengupta, A., and Pal, T.K. (2000). On comparing interval sets. Eur. J. Oper. Res., 127, 28-43. http://dx.doi.org/10.1016/S0377-2217(99) 00319-7

Sengupta, A., Pal, T.K., and Chakraborty, D. (2001). Interpretation of inequality constraints involving interval coefficients and a solution to interval linear programming. Fuzzy Sets Syst., 119, 129-138. http: //dx.doi.org/10.1016/S0165-0114(98)00407-2

Shen, J., Huang, G.H., An, C.J., Xin, X.Y., Huang, C., and Rosendahl, S. (2018). Removal of Tetrabromobisphenol A by Adsorption on Pinecone-Derived Activated Charcoals: Synchrotron FTIR, Kinetics and Surface Functionality Analyses. Bioresour. Technol., 247, 812-820. https://doi.org/10.1016/j.biortech.2017.09.177

Soyster, A.L. (1973). Technical note - Convex programming with setinclusive constraints and applications to inexact linear programming. Oper. Res., 21(5), 1154-1157. http://dx.doi.org/10.1287/opre. 21.5.1154

Steuer, R.E. (1981). Algorithms for linear programming problems with interval objective function coefficients. Math.Oper. Res., 6, 33-348. http://dx.doi.org/10.1287/moor.6.3.333

Tong, S.C. (1994). Interval set and fuzzy number linear programming. Fuzzy Sets Syst., 66, 301-306. http://dx.doi.org/10.1016/0165-0114 (94)90097-3

Wang, L., Huang, G.H., Wang, X.Q., and Zhu, H. (2018). Risk-based electric power system planning for climate change mitigation through multi-stage joint-probabilistic left-hand-side chance-constrained fractional programming: A Canadian case study. Renew. Sust. Energ. Rev., 82(1), 1056-1067. https://doi.org/10.1016/j.rser. 2017.09.098

Wang, L.Z., Huang, Y.F., Wang, L., and Wang, G.Q. (2014). Pollutant Flushing Characterizations of Stormwater Runoff and Their Correlation with Land Use in a Rapidly Urbanizing Watershed. J. Environ. Inf., 23(1), 44-54. http://dx. doi:10.3808/jei.201400257
Wu, C.B., Huang, G.H., Li, W., Xie, Y.L., and Xu, Y. (2015). Multistage stochastic inexact chance-constraint programming for an integrated biomass-municipal solid waste power supply management under uncertainty. Renew. Sustain. Energy Rev., 41, 1244-1254. http:// dx.doi.org/10.1016/j.rser.2014.09.019

Wu, C.B., Huang, G.H., Xin, B.G., and Chen, J.K. (2018). Scenario analysis of carbon emissions' anti-driving effect on Qingdao's energy structure adjustment with an optimization model, Part I: Carbon emissions peak value prediction. J. Clean. Prod., 172, 466474. https://doi.org/10.1016/j.jclepro.2017.10.216

Wu, S.M., Huang, G.H., and Guo, H.C. (1997). An interactive inexactfuzzy approach for multiobjective planning of water resource systems. Water Sci. Technol., 36(5), 235-242. http://dx.doi.org/10.1016/S 0273-1223(97)00479-4

Xu, Y., Huang, G.H., Cheng, G.H., Liu, Y. and Li ,Y. F. (2014). A TwoStage Fuzzy Chance-Constrained Model for Solid Waste Allocation Planning. J. Environ. Inf., 24(2), 101-110. http://dx. doi:10.3808/jei. 201400261

Xu, Y., Huang, G.H., and Shao, L.G. (2014). Agricultural farming planning and water resources management under fuzzy uncertainty. Eng. Optimiz., 46(2), 270-288. http://dx.doi.org/10.1080/0305215 X.2013.768239

Yan, X.P., Ma, X.F., Huang, G.H., and Wu, C.Z. (2010). An inexact transportation planning model for supporting vehicle emissions management. J. Environ. Inf., 15(2), 87-98. http://dx.doi.org/10.3808/j ei. 201000169

You, L., Li, Y.P., Huang, G.H., and Zhang, J.L. (2014). Modeling regional ecosystem development under uncertainty - A case study for New Binhai District of Tianjin. Ecol. Model., 288, 127-142. http:// dx.doi.org/10.1016/j.ecolmodel.2014.06.008

Zeng, G.M., Jiang, Y.M., Guo, H.C., and Huang, G.H. (2000). Two-dimensional numerical algorithm for water quality modeling in river systems with complicated topography. J. Environ. Sci., 12(4), 469473

Zhang, S.P., An, L.S., Shen, G.Q. and Niu, Y.G. (2014). Acoustic Pyrometry System for Environmental Protection in Power Plant Boilers. J. Environ. Inf., 23(2), 24-35. http://dx. doi:10.3808/jei. 201400265

Zhao, X., Chen, B., and Yang, Z.F. (2009). National water footprint in an input-output framework - A case study of China 2002. Ecol. Model., 220(2), 245-253. https://doi.org/10.1016/j.ecolmodel.2008.09. 016

Zhao, X., Yang, H., Yang, Z.F., Chen, B., and Qin, Y. (2010). Applying the input-output method to account for water footprint and virtual water trade in the Haihe River Basin in China. Environ. Sci. Technol., 44(23), 9150-9156. http://dx.doi.org/10.1021/es100886r

Zheng, Y.L., Nie, S.L., Ji, H., and Hu, Z. (2013). Application of a Fuzzy Programming through Stochastic Particle Swarm Optimization to Assessment of Filter Management Strategies in Fluid Power System Under Uncertainty. J. Optimiz. Theory Appl., 157(1), 276-286. http://dx.doi.org/10.1007/s10957-012-0152-0

Zhou, F., Guo, H.C., Chen, G.X., and Huang, G.H. (2008). The interval linear programming: a revisit. J. Environ. Inf., 11(1), 1-10. http://dx. doi.org/10.3808/jei.200800105 